\title{
Cell-lineage specificity of primary cilia during epididymis post-natal development
}

Agathe Bernet ${ }^{1}$, Alexandre Bastien ${ }^{2}$, Denis Soulet ${ }^{3}$, Olivia Jerczynski ${ }^{1}$, Christian Roy ${ }^{1}$, Maira Bianchi Rodrigues Alves ${ }^{1}$, Cynthia Lecours ${ }^{3,4}$, Marie-Ève Tremblay ${ }^{4}$, Janice Bailey ${ }^{2}$, Claude Robert ${ }^{2}$, Clémence

7 Belleannée ${ }^{1,5}$.

${ }^{1}$ Department of Obstetrics, Gynecology and Reproduction, Université Laval, CHU de Québec Research Center (CHUL), Quebec City, Quebec, Canada.

${ }^{2}$ Department of Animal Sciences, Université Laval, Quebec City, Quebec, Canada.

${ }^{3}$ Faculty of Pharmacy, Université Laval, CHU de Québec Research Center (CHUL), Quebec City, Quebec, 16 Canada.

${ }^{4}$ Department of Molecular Medicine, Université Laval, CHU de Québec Research Center (CHUL), Quebec 19 City, Quebec, Canada. 


\section{Abstract}

31 Primary cilia are sensory organelles that orchestrate major signaling pathways during organ

32 development and homeostasis. By using a double Arl13b/mCherry-Cetn2/GFP transgenic mouse

33 model, we characterized the spatio-temporal localization of primary cilia in the epididymis, from

34 birth to adulthood. We report here a constitutive localisation of primary cilia in peritubular

35 myoid cells and a dynamic profiling in differentiated epithelial cells throughout post-natal

36 development. While primary cilia are present at the apical pole of the undifferentiated epithelial

37 cells from birth to puberty, they are absent from the apical pole of the epithelium in adults,

38 where they appear exclusively associated with cytokeratin 5-positive basal cells. Exogenous

39 labeling of primary cilia marker Arl13b and IFT88 confirmed the cell lineage specific localization

40 of primary cilia in basal cells and myoid cells in human epididymides. From whole epididymis

41 tissues and serum-free cultures of DC2 murine epididymal principal cell lines we determined

42 that primary cilia from the epididymis are associated with the polycystic kidney disease-related

43 proteins polycystin 1 (PC1) and polycystin 2 (PC2), and Gli3 Hedgehog signaling transcription

44 factor. Thus, our findings unveil the existence of primary cilia sensory organelles, which have the

45 potential to mediate mechano/ chemo-signaling events in the epididymis. 


\section{Introduction}

Post-natal development (PND) of the epididymis is a multistep process synchronized

48 with an increase in androgen plasma level, arrival of testicular flow, and sperm release [1].

49 Proper epididymis PND is essential to generate a fully functional organ that will ensure optimal

50 post-testicular sperm maturation at the time of puberty (For review, [2]). Thus, identification of

51 cellular factors involved in the development of male reproductive functions is a compelling

52 research avenue that may help understand the aetiology of some unexplained male infertility 53 issues.

54 The adult epididymis is a single convoluted tubule divided into three to four main anatomical regions (i.e. initial segment in rodents, caput, corpus and cauda), each displaying

56 distinct morphological features. The lumen of the epididymis through which spermatozoa 57 transit is surrounded by a pseudostratified epithelium. The latter is mainly composed of 58 principal, clear and basal cells, whose functions are essential to ensure proper post-testicular 59 sperm maturation (for review [3]). The epididymis becomes fully functional following a series of 60 morphological changes that occur during its PND, particularly prior to puberty. These changes 61 include the formation and maintenance of a blood-epididymis barrier, the differentiation and 62 organization of epididymal cells to form a well-orchestrated pseudo-stratified epithelium, and 63 the regionalized expression of epididymal genes in the different segments of the organ.

The differentiation of epididymal cell populations and the establishment of distinct 66 humans [1, 4-11]. It consists of three main stages: the undifferentiated period, the period of 67 differentiation, and the period of expansion [12]. During the undifferentiated period $\left(1^{\text {st }}\right.$ week in 68 mouse), the epididymis epithelium is characterized by the presence of columnar cells that lack 69 stereocilia and form primitive junctions. The period of differentiation ( $2^{\text {nd }}$ to $5^{\text {th }}$ week in mouse) 70 consists in the appearance of differentiated and specialized cells, including clear, principal and 71 basal cells $[13,14]$; this period is associated with high cellular proliferation within the initial 72 segment. It has been proposed that the flow of testicular-derived fluid entering the epididymis 73 before sperm production may stimulate this proliferation/differentiation stage [1]. Finally, the 74 period of expansion $\left(6^{\text {th }}\right.$ to $8^{\text {th }}$ week in mouse) features the appearance of spermatozoa within 
75 the epididymal lumen, as well as an increase in the size of the epididymis, until cell division 76 arrest at around the $10^{\text {th }}$ week. Different factors such as luminal flow, lumicrine components

77 and androgens have been shown to participate in epididymis PND through epithelial cell 78 differentiation [15], [7, 16]; For review refer to [17]. However, the cellular mechanisms involved

79 in this process are unknown. The serendipitous finding of the presence of sensory primary cilia 80 organelles in the mammalian epididymis shed light on new potential developmental 81 mechanisms [18].

The primary cilium is a solitary cell extension that serves as a sensory organelle and a 83 signaling hub to control cell proliferation, migration, differentiation and planar polarity [19, 20]. 84 Thus, this biological antenna is required to ensure proper tissue development and homeostasis 85 and extends from the surface of most mammalian cells at the post-mitotic stage. Non-motile primary cilia are composed of a 9+0 axoneme (nine pairs of microtubules without central pair, in contrast to 9+2 motile cilia) and a basal body, which derives from the mother centriole of the centrosome. Depending on the biological system in which they are studied, primary cilia play two distinct roles: one as a signaling mediator through a variety of ciliary receptors and their downstream effector molecules, and the other as a mechanosensor through ion channels and transporter proteins. The signaling pathways coordinated by primary cilia are diverse and 92 include Hedgehog (Hh)[21], Wingless (Wnt/Notch) [22] and Platelet-derived growth factor 93 receptor (PDGFR) [23] signaling pathways. In addition to this function, some cells are responsive 94 to the shear stress exerted by the surrounding biological fluids through primary cilia 95 mechanosensing [24], for review [25]. For instance, primary cilia found at the surface of renal 96 intercalated cells, sense urine flow and control cell proliferation through ciliary Polycystin 1 97 (PC1), transient receptor potential channel interacting, and the Polycystin 2 (PC2) cation 98 channel[26]. Bending of the primary cilium triggered by flow within the nephron tubule induces 99 calcium influx via PC2 calcium transporter, and the downstream regulation of target gene 100 expression involved in cell proliferation [26].

Based on remarkable studies asserting the role of primary cilia in cell sensing, the 102 functional features associated with this organelle make it an ideal candidate for detecting and 103 transducing signals involved in organ development. Apart from the serendipitous observation of 
104 primary cilia in the epididymis of equine species reported by Arrighi in 2013 [18], no further

105 studies have investigated the characterization and potential role of these biological antennae in

106 epididymis development and homeostasis. In our study, we discovered for the first time the

107 presence of a primary cilia component in the human epididymis and portrayed their spatio-

108 temporal localization during epididymis PND in a double transgenic mouse model Arl13b-

109 mCherry/Centrin2-GFP [27]. Of relevance, impairment of primary cilia function is associated

110 with male infertility issues and other human diseases referred to as ciliopathies [28]. For

111 instance several reports indicate that Autosomal Polycystic Kidney Disease (APKD), a ciliopathy

112 affecting one in 800-1000 live birth, is associated with a higher prevalence of obstructive

113 azoospermia, increased epididymis and vas deferens volumes, and the presence of cysts in the

114 epididymis, seminal vesicles and prostate [29-31]. In addition, Von Hippel-Lindau Syndrome

115 (VHL), an autosomal dominant ciliopathy characterized by the predisposition for multiple

116 tumours, is associated with the development of epididymal cystadenomas that result in male

117 infertility when bilateral [32-35]. Considering that non-genetic environmental insults, such as

118 lithium treatment and folic acid uptake, have been shown to alter cilia length and functions [36-

119 40], portraying and unravelling the role of primary cilia during epididymis PND will open new

120 avenues concerning the diagnosis of unexplained male infertility cases. 


\section{Material and Methods}

\section{Human tissues and ethical consent}

123 Human epididymides were obtained from donors between 26 and 50 years of age through our 124 local organ transplantation program (Transplant Quebec, QC, Canada) after obtaining written

125 consent from the families. Experiments performed in our study were in compliance with the

126 Declaration of Helsinki and were conducted according to the policies for Human Studies with

127 ethical approval from the Centre Hospitalier Universitaire's (CHUQ) Institutional Review Board

128 (\#2018-4043). Human epididymides from three donors were processed as previously described

129 (ref [41]). In brief, the testicles were removed under artificial circulation to preserve tissues

130 assigned for transplantation and processed within $6 \mathrm{~h}$. The epididymides were dissected into

131 three segments, i.e. caput, corpus and cauda and fixed by immersion in paraformaldehyde $4 \%$

132 for immunohistological localization of Arl13b and IFT88.

134 Mouse tissues and ethics

135 Epididymides from C57BL/6 and $\mathrm{Tg}(\mathrm{CAG}-\mathrm{Arl} 13 \mathrm{~b} / \mathrm{mCherry}) 1 \mathrm{Kvand} \mathrm{Tg}$ (CAG-EGFP/CETN2)3-

$1364 \mathrm{Jgg} /$ KvandJ (referred to as Arl13b-Cetn2 tg in this study, Jackson Laboratory stock\# \#027967)

137 were used in this study. The Arl13b-Cetn2 tg double transgenic mice express both the ciliary 138 component ADP-ribosylation factor-like protein 13B (Arl13b) fused to the monomeric red

139 fluorescence protein mCherry and the centriolar protein Centrin2 (Cetn2) fused to GFP [27].

140 These mice were housed and reproduced in the elite animal facility of the CHU de Quebec

141 research Center. Animal experiments were approved by the ethical committee of the

142 Institutional Review Board of the Centre Hospitalier Universitaire de Québec (CHUQ)(CPAC

143 licenses 2016050 and 12016051, C. Belleannée) and were conducted in accordance with the

144 requirements defined by the Guide for the Care and Use of Laboratory Animals.

145 Epididymides were obtained from mice sacrificed at different post-natal ages. Post-natal day 1

146 (1 dpn) to $268 \mathrm{dpn}$ tissues were either 1) directly snap frozen in liquid nitrogen and stored at $147-80^{\circ} \mathrm{C}$ until use, 2) fixed with fixative containing $4 \%$ paraformaldehyde, $10 \mathrm{mM}$ sodium 148 periodate, $75 \mathrm{mM}$ lysine, and 5\% sucrose in $0.1 \mathrm{M}$ sodium phosphate buffer (PLP) by immersion 149 (1 to $28 \mathrm{dpn}$ ) or by perfusion via the left ventricle (28 to $56 \mathrm{dpn}$ ) as previously described [42], or 
150 3) fixed by intra-cardiac systemic fixation with $4 \%$ paraformaldehyde for transmission electron

151 microscopy (TEM) analysis as previously described and adapted [43].

152

\section{Cell culture}

154 Immortalized Distal Caput principal cells (DC2) developed from mouse tissues were kindly 155 provided by Marie-Claire Orgebin-Crist [44]. DC2 cell were maintained in Iscove's Modified

156 Dulbecco's Media (IMDM, Gibco, Invitrogen S.A.) containing $1 \mu$ M dihydrotestosterone (Fluka), $15710 \%$ of fetal bovine serum (FBS) (Gibco, Invitrogen S.A.) and $50 \mathrm{U} \mathrm{ml}^{-1}$ of penicillin $\mathrm{G}$ and

$15850 \mathrm{\mu g} \mathrm{ml}^{-1}$ streptomycin (Gibco, Invitrogen S.A.). Cells were kept in an incubator at $32,8{ }^{\circ} \mathrm{C}$ in $5 \%$ $159 \mathrm{CO}_{2}$ in air and $100 \%$ humidity. On the day before the experiment, $\mathrm{DC} 2$ cells were plated on 160 fibronectin treated coverslips in a 6-well plate at a density of a 125000 cells per well. Cells were 161 starved from serum overnight prior to being fixed and used for immunofluorescence staining.

\section{Immunofluorescence staining}

164 Epididymides from Arl13b-Cetn2 tg mice fixed with PLP were treated for immunofluorescence 165 as previously described [45]. In brief, after three washes in PBS, tissues were cryoprotected with $16630 \%$ sucrose in PBS for several hours at $4^{\circ} \mathrm{C}$, embedded in Tissue-Tek ${ }^{\circledR}$ O.C.T. Compound 167 (Sakura ${ }^{\circledR}$ Finetek, USA), and quick-frozen. Five to $25-\mu$ m-thick sections were cut on a cryostat 168 (Shandon Cryotome, Thermo) and collected onto Superfrost/Plus slides (Superfrost 169 Fisherbrand $\mathrm{d}^{\mathrm{TM}}$ ). For indirect immunofluorescence staining, sections were hydrated for $15 \mathrm{~min}$ in 170 PBS and treated for 4 min with 1\% Sodium Dodecyl Sulfate (SDS) and 2\% Triton X-100 in PBS. 171 Sections were washed in PBS for 5 min and then blocked in PBS containing 1\% BSA for 15 min. 172 Sections were then incubated overnight in a humid chamber at $4^{\circ} \mathrm{C}$ with primary antibodies 173 diluted in DAKO solution (DAKO Corp., Carpinteria, CA) and directed against specific markers of 174 basal, principal, clear and myoid cells as well as primary cilia components (Table 1). When 175 specified, an antigen retrieval step for $10 \mathrm{~min}$ at $110^{\circ} \mathrm{C}$ in citrate buffer was included prior to 176 incubation with the primary antibodies. Sections were washed in high-salt PBS (2.7\% NaCl) twice 177 for $5 \mathrm{~min}$ and once in normal PBS. Respective secondary antibodies were then applied for $1 \mathrm{~h}$ at 178 room temperature followed by washes, as described above. Characteristics of these antibodies 
179 are described in Table 1 . Slides were mounted in Vectashield medium containing $4^{\prime}, 6^{\prime}$ -

180 diamidino-2-phenylindole (DAPI; Vector Laboratories, Inc., Burlingame, CA) for imaging.

181 For Immunofluorescence assays performed on cells, the latter were plated on fibronectin

182 coated slides at a density of 125000 cells per well and fixed with 4\% PFA for 10 min. After

183 washes with PBS, blocking was performed for 30 min in PBS solution with 1\% BSA and $0.1 \%$

184 Triton X-100. Slides were incubated for $1 \mathrm{hr}$. with primary antibody (1:400) followed by washes,

185 and for 1 hour with the appropriate secondary antibody (1:800). Slides were mounted in

186 Vectashield medium, as described above.

\section{Confocal imaging}

189 Digital images were acquired by confocal microscopy on an inverted Olympus IX80 microscope

190 equipped with a WaveFX-Borealin-SC Yokagawa spinning disk (Quorum Technologies; CFI 191 equipment to SE) and an Orca Flash4.0 camera (Hamamatsu). Image acquisition was performed

192 using Metamorph software (Molecular Devices). Optical Z-sections were acquired for each 193 channel and projected into a single picture using ImageJ. Low (20x) and high (100x)

194 magnification pictures were taken for the three major epididymis segments, the caput, corpus 195 and cauda epididymis.

196 Three-dimensional animations were generated with Bitplane Imaris software v7.5 (Bitplane, 197 Zurich, Switzerland) using images acquired by an Olympus FV-1000 confocal microscope

198 (Olympus Canada; CFI equipment to DS) equipped with a PLAPON60XOSC objective lens (NA 199 1.4). Isosurfaces were generated in the surpass module, and the camera was rotated to show 200 structures of interest. Generated isosurface renderings were rotated, panned, and zoomed 201 while recording the animation.

202 To determine primary cilia features throughout the epididymis, images were acquired on a Zeiss 203 LSM700 confocal microscope with a 40x/0.95 plan-apo lens. The resolution was set to the 204 highest possible according to the lens in use $(0.145 \mu \mathrm{m} /$ pixel). Whole epididymal sections (25 $205 \mu \mathrm{m}$ of thickness) were imaged in the z-axis, generally 15-20 $\mu \mathrm{m}$ at $1 \mu \mathrm{m}$ interval and large 206 regions of at least $1 \mathrm{~mm}^{2}$ were imaged in the $\mathrm{XY}$ plane with mosaic tiling at $10 \%$ overlap. 207 Excitation was done with lasers at 405, 488 and $555 \mathrm{~nm}$ while emission was selected with a 
208 variable dichroic splitter. Large data processing was done with custom Image [46] macro and 209 Matlab code. Briefly, images were converted from a single CZI mosaic file to multiple TIFFs. Each

210 tile was then compressed along the z-axis using a maximum intensity projection. Tiles were then

211 stitched together using MIST [47]. Gaussian filtering, thresholding and skeletonizing were

212 performed on the eGFP channel to outline distinct tubules. Centrosomes and cilia were 213 detected using ImageJ's Find Maxima. The centrosome list was filtered in Matlab to keep only 214 the closest to each cilium and cilia without visible centrosome were excluded. We determined 215 the absolute angle of each cilium with a rotating pattern in order to obtain a maximum at the 216 cilium angle relatively to the closest tubule edge. Matlab and ImageJ macro to process cilia 217 orientation from epididymal confocal acquisitions are available on

\section{8 https://github.com/alexandrebastien/Cilia.}

\section{Immunohistochemistry (IHC)}

221 Fixed epididymides from C57BL/6 mice were treated for IHC as previously described [48]. In 222 brief, tissues were dehydrated, embedded in paraffin and stored at $4^{\circ} \mathrm{C}$ until use. Paraffin 223 sections (5 $\mu \mathrm{m}$ thickness) were deparaffinised in toluene, hydrated, and then, where specified, 224 treated for antigen retrieval with citrate buffer $(\mathrm{pH}$ 6.0). Endogenous peroxidase activity was 225 then quenched with $3 \% \mathrm{H}_{2} \mathrm{O}_{2}(\mathrm{v} / \mathrm{v})$ in methanol for $10 \mathrm{~min}$. Sections were washed for 5 min in $22695 \%$ ethanol and $5 \mathrm{~min}$ in PBS. Non-specific binding sites were blocked with 0.5\% BSA for $1 \mathrm{~h}$. 227 Primary antibodies diluted in DAKO were applied overnight at $4^{\circ} \mathrm{C}$ (Table 1). For control 228 sections, PBS replaced the primary antibodies. Sections were subsequently incubated with 229 biotinylated 1) donkey anti-goat or 2) goat anti-rabbit antibodies for $60 \mathrm{~min}$, and with ABC elite 230 reagent (Vector Laboratories, Inc. Burlingame, Ca) for $30 \mathrm{~min}$. Immunostaining was revealed 231 using 3-amino-9-ethylcarbazole (AEC). Mayer's hematoxylin solution was used for 232 counterstaining, and mounted under cover slips using an aqueous mounting medium (Sigma). 233 Slides were observed under a Zeiss Axioskop 2 Plus microscope linked to a digital camera from 234 Qimaging. Images were captured using the QCapture Pro (Qimaging Instruments). 
238 Mice were anesthetized with ketamine (intraperitoneally) and tissues were fixed by intracardiac 239 perfusion with PBS for 2 min followed by 4\% PFA for 2 min and treated as previously described 240 and adapted [43]. In brief, tissues were further fixed by immersion for $2 \mathrm{~h}$ in $3.5 \%$ acrolein, 241 followed by $3 \mathrm{~h}$ in 4\% PFA and washed three times in PBS for 15 min. Fifty-micrometer-thick 242 transverse sections of the epididymis were cut in PBS using a vibratome (Leica VT100S) and 243 stored at $-20^{\circ} \mathrm{C}$ in cryoprotectant until further processing.

\section{Electron microscopy}

246 Sections stored in cryoprotectant were rinsed in PBS, postfixed in 1\% osmium tetroxide for 30 247 min at room temperature and dehydrated in ascending concentrations of ethanol (from 35\% to 248 100\%). Sections were then treated with propylene oxide and impregnated in Durcupan resin 249 (EMS) overnight at room temperature. After mounting between ACLAR embedding films (EMS), 250 the resin was polymerized at $55^{\circ} \mathrm{C}$ for $72 \mathrm{~h}$. Areas of interest were excised from the embedding 251 films, re-embedded at the tip of resin blocks, and cut at $65-80 \mathrm{~nm}$ of thickness using an 252 ultramicrotome (Leica Ultracut UC7). Ultrathin sections were collected on square mesh grids 253 (EMS) and examined at $80 \mathrm{kV}$ with an FEI Tecnai Spirit G2 transmission electron microscope. 254 Pictures were acquired using an Orca-HR camera (10 MP; Hamamatsu). 
Results

Primary cilia extend from basal and smooth muscle cells in the epididymis of adult mice

259 Due to the fact that primary cilia are small, dynamic and present as a solitary figure on the cell 260 surface, these organelles are difficult to detect within in situ or in vivo whole organ systems. We 261 used the Arl13b-Cetn2 tg mouse model [27] to accurately study lineage specificity of primary 262 cilia from whole epididymis tissues. Arl13b-positive structures were observed within the 263 epithelium and in the surrounding smooth muscle layer of the epididymis (Fig. 1A). The 264 microtubule component and primary cilia marker acetylated tubulin (Ac-Tub) was observed 265 associated with the sperm flagellum and co-localized with Arl13b-positive cell extension, further 266 confirming the specific detection of non motile primary cilia from Arl13b-Cetn2 tg mice (Fig. 1BC). By using specific markers of distinct epididymis epithelial cell populations (i.e. keratin 5 for basal cells, aquaporin 9 for principal cells and alpha actin for smooth muscle cells) we determined the cell-specific localization of primary cilia from adult Arl13b-Cetn2 tg mice (Fig. 2). As evidenced by labelling with the alpha actin smooth muscle cell marker, primary cilia were found in the peritubular area associated with smooth muscle cell centriole (Fig. 2A, inset). The 272 ultrastructure of primary cilia in smooth muscle cells is composed of a basal centriole and an 273 axoneme extension as observed by transmission electron microscopy (Fig. 2B). In addition, 274 Arl13b-positive primary cilia were never observed associated with aquaporin 9-positive principal 275 cells, nor aquaporin 9-negative clear cells from adult mice (Fig. 2C). Finally, we found that 276 primary cilia extended from the centriole of keratin 5-positive basal cells (Fig. 2D, inset). From 277 the whole image acquisitions performed on adult epididymis tissues, all basal cells that were 278 observed within a $25 \mu \mathrm{m}$ tissue section exposed a primary cilium. While the major part of the 279 basal cell body is located at the base of the epithelium, basal cells dynamically extend towards 280 the lumen of the epididymis through axiopodia [49, 50]. According to basal cell 3D 281 reconstruction from transgenic epididymal tissues, we observed that primary cilia take their 282 origin from the centriole located at the apical pole of the cell body and at the base of the 283 axopodia (Fig. 3). In addition, basal cell-associated primary cilia never cross epididymis tight 284 junctions as evidenced by 3D reconstruction after ZO-1 staining (Supplementary file 1). 


\section{Primary cilia profiling along the adult epididymis}

287 The epididymis is a highly segmented tubule with each segment displaying distinct features in 288 terms of cell populations, cell composition and cell functions [51]. We investigated the 289 properties of basal cell primary cilia throughout the different segments of the epididymis from 290 Arl13b-Cetn2 tg mice (i.e. initial segment (IS), caput, corpus and cauda) by whole tissue confocal 291 imaging and digital image processing (Fig. 4). Primary cilia were observed associated with 292 peritubular myoid and basal cells in all segments of the epididymis, with a higher proportion in 293 the basal cells from the distal regions (Fig. 4A, Supplementary file 2). In the IS basal cell primary 294 cilia appeared as short 2-3 $\mu \mathrm{m}$ extensions directing towards the base of the epithelium, 295 according to GFP-positive centriole localization (Figs. 4A, B). In the other segments, i.e the 296 caput, corpus and cauda, primary cilia appeared as longer 5-10 $\mu \mathrm{m}$ extensions, presenting with 297 erratic directions. Furthermore, myoid cell primary cilia presented no obvious variation from 298 one segment to another in terms of length $(5-6 \mu \mathrm{m})$ or orientation (generally perpendicular to 299 the intratubular axis). In addition to their localization throughout the basal and peritubular 300 myoid cells of the epididymis, primary cilia were also associated with basal cells in the vas 301 deferens (VD) (Fig. 4A, VD) and were observed as tremendously long extensions in the efferent 302 ducts (ED) (Fig. 4A, ED). In ED, ciliated cells presenting with numerous centrin2-positive 303 centrioles were observed from the apical surface of the ducts. Moreover, elongated Arl13b304 positive primary cilia $(15-40 \mu \mathrm{m})$ were found associated with centrioles located in non-ciliated 305 cells of the ED.

\section{Dynamic primary cilia exposure during epididymis post-natal development}

308 The epithelium of the epididymis undergoes important changes during the different PND stages

309 [1]. We explored the dynamics of primary cilia exposure that accompanies epididymis 310 epithelium development just after birth (at $5 \mathrm{dpn}$ ), at pre- and post-pubertal ages (at 30 and 42 $311 \mathrm{dpn}$, respectively), and at the adult stage (at 42 to $268 \mathrm{dpn}$ ) (Fig. 5). The Arl13b-Cetn2 tg mouse 312 model displays the same pattern of epididymis development as previously described in wild313 type mice [12] (Fig. 5, Top). For instance, H \& E staining performed on the epididymis at 5 dpn 314 stage shows undifferentiated columnar cells of the epididymal epithelium surrounding an empty 
315 lumen. At 30 dpn, we observed a pseudo-stratified epithelium and the presence of basal cells.

316 The lumen is filled with non-cellular material. At $42 \mathrm{dpn}$ and $268 \mathrm{dpn}$, the pseudostratified

317 epithelium surrounds large lumens filled with spermatozoa. At an early stage of dpn (Fig. 5, at 5

318 dpn Bottom and supplementary file 3), primary cilia are observed by confocal microscopy as

319 short Arl13b-positive extensions associated with centrioles, located at the apical surface of non-

320 differentiated columnar cells. Primary cilia extensions are extensively observed associated with

321 myoid cells surrounding the epithelium. At $30 \mathrm{dpn}$, prior to puberty, primary cilia are found at

322 the apical surface of the epithelium associated with differentiated cells, potential clear and/or

323 principal cells, and associated with basal cells. After puberty, at $42 \mathrm{dpn}$ (Fig. 5, at 42 dpn and

324 supplementary file 4) and $268 \mathrm{dpn}$, primary cilia extensions are exclusively found in basal cells

325 within the epithelium as well as in surrounding myoid cells. Thus, according to the PND stage of

326 the mouse epididymis, primary cilia are localized at different positions along the epididymal

327 epithelium, i.e. facing the lumen during the undifferentiated period, facing the lumen and at the

328 base of the epithelium during the period of differentiation, and exclusively at the base of the

329 epithelium during the expansion phase. Primary cilia are observed in myoid cells at all PND

330 stages studied. According to ZO-1 immunostaining of the apical pole of the epididymis (Fig. 6),

331 primary cilia extend from the epithelial cell surface at an early stage of PND (at 5 dpn) and are in

332 direct contact with the intraluminal compartment.

334 Primary cilia of the epididymis are potential mechano/chemo-sensors of the cell-surrounding

\section{5 environment}

336 According to the literature that describes the function of primary cilia in different model 337 systems, two major roles are associated with these biological antennae: the first as a chemo338 sensor of major signaling pathways (e.g. Hg, Wnt, PDGFR), and the second as a mechano-sensor 339 responsive to shear-stress and pressure exerted on the cell surface by extracellular bodily fluid.

340 By analogy with other tissues, we investigated the functional signaling components associated 341 with primary cilia in the epididymis.

342 We first explored the expression level of PC1 and PC2 (Fig. 7), two major players in the primary 343 cilia-dependent fluid-flow mechanosensation. At dpn 7, PC1 was detected at the apical pole of 
344 undifferentiated cells as well as in peritubular myoid cells in the different epididymal segments

345 (Fig. 7A). At this post-natal stage, a stronger signal was observed in the vas deferens (Fig. 7A,

346 VD). In tissues from adult mice, PC1 was intensively detected in the cytoplasm and the apical

347 pole of the epithelium in the initial segment/caput of the epididymis. While PC1 intensity

348 transitionally decreased in the corpus compared to the caput, it drastically increased in the

349 apical membrane of epithelial cells from the cauda as well as in the vas deferens (VD). PC1 was

350 also detected, but to a lower extent, in the peritubular myoid cells. While PC2 could not be

351 detected on epididymal sections by IHC under our experimental conditions (not shown), we

352 observed a co-localisation between PC2 and the primary cilia marker Ac-Tub in the DC2

353 epididymal principal cell line (Fig.7B, arrows).

354 We next investigated the expression of GLI3, a downstream transcriptional factor of the Hh

355 pathway (Fig. 8). In adult epididymis, GLI3 was observed as discontinuous staining at the apical

356 pole of the epithelium in the corpus and cauda epididymidis. In addition, strong GLI3 staining

357 was observed in the nucleus of different epithelial cell populations in all segments of the organ.

358 This staining was particularly intense in basal cell nuclei (Fig. 8, stars and insets).

360 Presence of primary cilia components in the human epididymis

361 In order to translate our observations made from a transgenic mouse model, we investigated

362 the expression of two primary cilia markers, Arl13b and IFT88, in human epididymal tissues (Fig.

363 9, Supplementary file 5). We observed strong exogenous staining for Arl13b in the apical pole

364 of the epithelial cells from the proximal segment, corresponding to the efferent ducts/caput

365 (Fig. 9, ED/caput inset). Whereas the signal was less intense in the corpus and cauda 366 epididymidis, it was mainly localized in peritubular myoid cells as well as in basal cells (Fig. 9, 367 arrows in insets). An identical detection profile was observed for IFT88 (Supplementary file 5). 


\section{Discussion}

370 Primary cilia are solitary antennae found at the cell surface and are fundamental to organ

371 development and homeostasis [20]. Accordingly, impairment of primary cilia formation in

372 humans is responsible for a broad range of clinical syndromes-including male infertility-and

373 diseases referred to as ciliopathies [28-35]. While these organelles have been broadly studied in

374 most biological systems, investigations performed on primary cilia in the male reproductive

375 system remain scarce $[18,52]$. Acknowledging the potential of primary cilia in the diagnosis of

376 unexplained male infertility, we portrayed for the first time the cell lineage specificity of primary

377 cilia in the epididymis, the organ of the male reproductive system in charge of post-testicular

378 sperm maturation (i.e. acquisition of motility and abilities to recognize and fertilize an egg) and

379 sustaining male fertility.

380 Almost every vertebrate cell has a primary cilium, which is a dynamic cell surface projection

381 playing the role of a sensory and signaling organelle. Although primary cilia were first described

382 by Ecker in 1844, knowledge of the full scope of their functions is continuously expanding with

383 the development of transgenic mouse models and the increasing number of human diseases

384 related to primary cilia dysfunctions [53]. With the re-appreciation of this biological antenna

385 over the past 10 years, it has become clear that the role of primary cilia in sensing the

386 extracellular environment potentially takes place in almost every cell responsive to intercellular

387 cross-talks and bodily fluid changes. The epididymis is a singular tubule that comprises distinct

388 segments, each controlling the composition of a dynamic epididymal fluid for proper sperm

389 maturation. Several mechanisms of intercellular communication between epididymal somatic

390 cells and between somatic and spermatic cells have been shown to be of major importance for

391 the control of sperm fertilizing abilities and reproductive outcomes [45, 50, 54-56]. We unveiled

392 here, for the first time, the existence of primary cilia displaying a cell lineage specificity and key

393 features of signaling antenna in the developing epididymis. In light of the spatio-temporal

394 changes of primary cilia observed during PND, and the functional components associated with

395 these antenna (i.e. PC1/PC2 and Gli3), we propose a novel mechanism of intercellular

396 communication involving primary cilia organelles in the control of epididymis development and

397 homeostasis (Fig. 10). 
398 By using a transgenic mouse model developed to detect endogenous fluorescence in both ciliary extension and centrioles, we determined that primary cilia were exclusively associated with 400 basal cells in the epididymal epithelium of adult mice. This is consistent with the serendipitous 401 observation made in 2013 by Arrighi, who documented the presence of primary cilia in the 402 epididymis of equine species [18]. In this unique article on epididymis primary cilia, typical 9+0 403 microtubular patterns and ciliary extensions were observed by transmission electron 404 microscopy associated with basal cells, according to their characteristic morphological features. 405 Our findings not only confirmed the cell specificity of primary cilia though co-localization of 406 primary cilia with keratin-5 positive basal cells, but also underscored a change in ciliary features 407 (length, orientation and signaling components) throughout the different segments of the 408 epididymis. Epididymal basal cells present with dynamic axiopodia extensions that cross the 409 blood-epididymis barrier to sense the lumen $[49,50]$. According to our observations, the fact 410 that primary cilia never cross the apical tight junctions in adult epididymis - even when basal 411 cells present with elongated axiopodia-suggests that primary cilia might sense intra-epithelial 412 extracellular fluid rather than the intraluminal fluid in adult mice.

413 Basal cells from several other stratified and pseudo-stratified epithelia expose primary cilia with 414 distinct signaling functions. For instance, basal cells from the olfactory epithelium have primary 415 cilia that control basal cell activation, proliferation and differentiation, and as such are 416 necessary for regeneration of the epithelium following injury [57]. In addition, primary cilia 417 associated with basal cells from the mammary gland are involved in the acquisition of their stem 418 cell properties via Hh signaling, thereby controlling mammary tissue outgrowth and basal cell 419 tumor development [58]. While limited information is available with regard to basal cell 420 functions in the epididymis, their association with primary cilia could underlie a new functional 421 hypothesis related to epididymis development and homeostasis. According to gene expression 422 profiling, basal cells share common properties with adult stem cells and were recently proposed 423 to differentiate into columnar cells as a mechanism of epididymal epithelium regeneration [59]. 424 Although basal cells from the epididymis do not seem to be progenitors of the other cell 425 populations during early development $[14,60]$, they may participate in epithelium regeneration 426 via primary cilia signaling, in a similar manner to other epithelia. In addition, primary cilia- 
427 dependent control of tumorigenesis via Hh signaling appears to be common in cases of basal 428 cell carcinoma [61]. It is striking that the epididymis rarely develops tumors [62, 63]. Among the 429 few cases that have been described in the literature, two thirds of epididymal epithelial tumours 430 (papillary cystadenoma) are associated with Von Hippel-Lindau (VHL) syndrome, an atypical 431 ciliopathy[64]. Thus, it is possible that primary cilia monitor the epithelium stability of the 432 epididymis and prevent oncogenic events through $\mathrm{Hh}$ or Wnt signaling pathways, whose 433 downstream effectors are enriched in basal cells ([59] and our data).

434 Depending on the physiological context, primary cilia could exert dual sensory functions, either 435 as mechanosensors and/or chemosensors (For reviews, [19, 20, 65]). With regard to 436 chemosensing, the existence of transition fibers located at the base of the cilium physically 437 confines specialized molecules into the cilium, which potentiates signaling responses to 438 extracellular cues. Among these signals, primary cilia coordinate Hh, Wnt and PDGFR and signal439 transduction machineries. Interestingly, the components of these pathways-either agonists or 440 downstrean effectors - are all found enriched in basal cells of the epididymis [59] and are 441 particularly relevant to the control of epididymis homeostasis and post-testicular maturation 442 events occurring in this organ [62, 66-71]. For instance, blockage of the Hh signaling pathway 443 following cyclopamine administration triggers a significant decrease in Gli1 and Gli3 444 transcriptional factor expression as well as a significant decrease in epididymal sperm motility 445 [69]. Whether or not this phenotype relies on primary cilia-dependent Hh signaling remains to 446 be established. Complementary in vivo studies on basal cell primary cilia will further define the 447 contribution of this organelle to epididymis physiology, and ultimately to sperm maturation and 448 male fertility.

449 Finally, the fact that primary cilia are observed at the apical pole of the epithelium from early 450 stages of PND to puberty and colocalize with polycystins (PC1 and PC2), suggests that these 451 organelles might play the role of mechanosensors before puberty. This type of primary cilia452 dependent mechanosignaling is broadly documented in the kidney, which shares the same 453 embryonic origin as the epididymis and presents numerous functional similarities with it [24, 454 26]. In epithelial cells from the distal convoluted and connecting tubules of the kidney, the flow 455 shear stress exerted at the surface of the cells is sensed by primary cilia exposed at the cell 
456 surface. The subsequent physical bending of primary cilia triggers PC1 dependent-transepithelial 457 transport of calcium and controls tissue morphogenesis [26]. Approximately 40 years ago, Sun 458 and Flickinger proposed that the flow of testicular-derived fluid entering the epididymis before 459 sperm production may stimulate epithelial cell proliferation and differentiation in the 460 epididymis [1]. Building on our new findings, it is now conceivable that primary cilia are 461 strategically positioned in direct contact with the lumen of the epididymis to initiate shear-flow 462 dependent epithelium maturation and sense the first wave of spermatozoa at puberty to 463 sustain maturation.

464 In conclusion, our study reveals for the first time the cell lineage specificity of primary cilia along 465 the epididymis at different stages of PND. Acknowledging the important role played by these 466 sensory organelles in most biological systems, our work opens new avenues of research 467 concerning the cellular control of epididymal development and homeostasis. Importantly, since 468 primary cilia components are conserved in the human epididymis, current drugs controlling 469 ciliary functions might constitute new targets for the development of non-hormonal male 470 contraceptives or treatments for cases of unexplained male infertility.

\section{Acknowledgements}

473 We thank Pr Sylvie Breton, Ph.D., for providing aquaporin 9 antibody used in this project, as well 474 as Pr Marie-Claire Orgebin-Crist, Ph.D., for providing epididymal DC2 cell line. We would like to 475 acknowledge the contribution of Sabine Elowe, Ph.D., for confocal imaging support, Robert 476 Sullivan, Ph.D., for giving us access to the biobank of human tissues, and Christine Légaré, M.Sc., 477 for her technical support. The contributions of Johanne Ouellet in histology services, France 478 Couture in illustration and Julie-Christine Lévesque, M.Sc., from the bio-imagery core facility are 479 also acknowledged. Enthusiastic informal discussions with Rex Ress, Ph.D. were highly 480 appreciated throughout this project.

\section{Competing interests}

483 The authors declare no competing or financial interests. 


\section{Author contributions}

486 Experiments were conceived and designed by $A B a, A B e, C B, C R o y$, and $D S$. Contributed to

487 critical reagent, resources and expertise; CB, CRo, DS, JB, MET. Experiments were performed by $488 \mathrm{ABa}, \mathrm{ABe}, \mathrm{CB}, \mathrm{CL}, \mathrm{CRoy}, \mathrm{DS}, \mathrm{OJ}, \mathrm{MBR}$. Data were processed and analyzed by $\mathrm{ABa}, \mathrm{ABe}, \mathrm{CB}, \mathrm{CRoy}$, 489 DS, MBR, and supervised by $C B, C R o, D S, J B$, and MET. The manuscript was written by $A B e, C R o y$ 490 and CB, and critically reviewed by all authors.

492 Funding support

493 This work was supported by a NSERC operating grant (RGPIN-2015-109194) and a FRQS-Junior 1 494 salary award to CB, NSERC grant to MET (RGPIN-2014-05308), NSERC grant to CRo (RGPIN-2017495 04775), and a FRQS-Junior 2 career award and CFI equipment to DS. ABe is recipient of a CRDSI 496 fellowship. MBR is recipient of a FAPESP fellowship. CL is recipient of a FRQS master award 497 scholarship. MET is a Canada Research Chair (Tier 2) in Neuroimmune Plasticity in Health and 498 Therapy. 
501 Fig. 1. Detection of primary cilia in Arl13b-Cetn2 tg mice. Epididymal sections from Arl13b502 Cetn2 tg mice (A) were stained for the primary cilia marker acetylated tubulin (Ac-Tub) (B). 503 Arl13b-positive cilia extensions colocalize with Ac-Tub (Arrows, A, B and C), which validates this 504 mouse model for the study of primary cilia in the epididymis. Ep.: epithelium; Lu.: lumen; Mc.: 505 myoid cells. Scale bar: $10 \mu \mathrm{m}$.

Fig. 2. Primary cilia extend from basal and peritubular myoid cells in the epididymis of adult mice. Immunofluorescent staining for alpha-actin myoid cell marker (A and inset), aquaporin 9 (Aqp9) principal cell marker (C), keratin-5 (Krt5) basal cell marker (D and inset) was performed on epididymal sections from Arl13b-Cetn2 dTg mice. Primary cilia structural components were detected by transmission electron microscopy in epididymal peritubular myoid cells from C57BL/6 mice (B). Ax.: axoneme; Bb.: basal body; Ct.: connective tissue; Lu.: lumen; Mc.: myoid 513 cells.

Fig. 3. Primary cilia extend from basal cells' main body. Confocal acquisition of the initial 516 segment from the epididymis of Arl13b-Cetn2 dTg mice showing a ciliated basal cell. Arl13b517 positive primary cilia extend from centrin2-positive centrioles located at the base of the 518 axiopodia (Axp.).

520 Fig. 4. Primary cilia are observed in the efferent ducts (ED), the vas deferens (VD), and the 521 different segments of the mouse epididymis. Primary cilia were observed associated with basal 522 cells (stars) and peritubular myoid cells in all segments of the epididymis (A). The angle of basal 523 cell primary cilia relative to the closest tubule edge was determined by image processing in each 524 epididymal region, i.e. IS/Caput ( $\mathrm{n}=899$ cells), corpus ( $\mathrm{n}=371$ cells) and cauda ( $\mathrm{n}=1065$ cells) from 5253 mice. Proportions of primary cilia with distinct angles were distributed between $-90^{\circ}$ for cilia 526 directed towards the base of the epithelium to $90^{\circ}$ for cilia directed towards the lumen (B). Ep.: 527 epithelium. Lu.: Lumen. Mc.: myoid cell. IS: initial segment. Scale bars: $10 \mu \mathrm{m}$. 
529 Fig. 5. Primary cilia are observed at different stages of epididymis post-natal development.

530 Hematoxylin and eosin staining (H \& E) and confocal imaging were performed on epididymis

531 sections from Arl13b-Cetn2 dTg mice at different stages of post-natal development, i.e. at 5, 30,

53242 and $268 \mathrm{dpn}$. Schematic representation of primary cilia location is shown throughout post-

533 natal development with centrioles displayed in green and primary cilia in red. NDC: non-

534 differentiated cells; DC: differentiated cells; MC: myoid cells; Lu.: lumen.

535

536 Fig. 6. Primary cilia are in contact with the intraluminal compartment of the epididymis at an early stage of post-natal development. Immunofluorescent staining for the tight junction

538 protein-1 (ZO1) on the cauda epididymidis of Arl13b-Cetn2 dTg mice at 5 dpn. Lu.:Iumen; 539 Ep.:epithelium.

541 Fig. 7. Expression of Polycystin1 (PC1) and Polycystin 2 (PC2) in epididymal cells and in the

542 mouse epididymis. (A) Immunohistochemical staining for PC1 on the epididymis of Arl13b543 Cetn2 dTg mice at 7 and 40 dpn. Cap.: caput; Cor.:corpus; Cau.:cauda; VD: Vas deferens. Scale 544 bar: $50 \mu \mathrm{m}$. (B) Immunofluorescent staining for PC2 and the primary cilia marker acetylated 545 tubulin (Ac-Tub) in DC2 cells.

546

547 Fig. 8. Expression of Gli3 in the mouse epididymis. Immunohistochemical staining for Gli3 on 548 the epididymis of Arl13b-Cetn2 dTg mice at 40 dpn. P-Cap: proximal caput; D-Cap: distal caput; 549 Cor.:corpus; Cau.:cauda. Inset scale bar: $10 \mu \mathrm{m}$

551 Fig. 9. Expression of Arl13b in the human epididymis. Immunohistochemical staining for Arl13b 552 was performed on the different segments of a human epididymis (donor age $=50$ ).

554 Fig. 10. Cell lineage specificity and potential roles of primary cilia in the epididymis during 555 postnatal development. Potential mechano/chemo-sensory functions associated with epithelial 556 cells primary cilia are presented. 


\section{Supplementary files.}

558 Supplementary file 1. 3D reconstruction of the epididymal epithelium after ZO-1 staining in

559 adult Arl13b-Cetn2 tg mice

560 Supplementary file 2. Proportion of primary cilia found in basal and myoid cells from different

561 epididymal segments.

562 Supplementary file 3. 3D reconstruction of the epididymal epithelium in Arl13b-Cetn2 tg mice 563 at early stage of PND (at $5 \mathrm{dpn}$ ).

564 Supplementary file 4. 3D reconstruction of the epididymal epithelium in Arl13b-Cetn2 tg mice 565 after puberty (at $40 \mathrm{dpn}$ ).

566 Supplementary file 5. Immunohistochemistry staining for IFT88 in human epididymis.

569 Table 1. List of antibodies used in our study.

\begin{tabular}{|r|c|c|c|c|c|c|}
\hline Type & Name & Host species & Dilution & Antigen retrieval IHC & Company & Catalog number \\
\hline polyclonal & Cytokeratin 5 & rabbit & $1-250$ & no & Abcam & ab53121 \\
\hline polyclonal & AQP9 & rabbit & $1-500$ & no & N/A & N/A \\
\hline monoclonal & ZO-1 & mouse & $1-100$ & no & Thermofisher & ZO1-1A12 \\
\hline polyclonal & $\alpha$-actin & rabbit & $1-100$ & no & Abcam & ab5694 \\
\hline polyclonal & Arl13b & rabbit & $1-50$ & no & Proteintech & $17711-1-A P$ \\
\hline monoclonal & Acetylated tubulin & mouse & $1-250$ & no & Sigma & T7451 \\
\hline polyclonal & IFT88 & rabbit & $1-200$ & no & Proteintech & $13967-1-A P$ \\
\hline polyclonal & PC1 & rabbit & $1-100$ & no & Bioss & $2157 R$ \\
\hline polyclonal & PC2 & rabbit & $1-400$ & no & Abcam & ab53281 \\
\hline monoclonal & Shh & rabbit & $1-500$ & citrate buffer & Abcam & ab52919 \\
\hline monoclonal & Ihh & rabbit & $1-100$ & no & Abres \\
\hline
\end{tabular}


584

585

586

587

588

589

590

591

592

593

594

595

596

597

598

599

600

601

602

603

604

605

606

607

608

609

610

611

612

613

614

615

616

617

618

619

620

621

622

623

624

625

626

627

628

629

\section{References.}

1. Sun, E.L., et al.,Development of cell types and of regional differences in the postnatal rat epididymis. Am J Anat, 1979. 154(1): p. 27-55.

2. Rodriguez, C.M., et al., The development of the epididymis, in The epididymis: from molecules to clinical practices, R.a. Hinton, Editor. 2002, Kluwer Academic/Plenum Publishers. p. 251-267.

3. Belleannée, C., et al.,Region-specific gene expression in the epididymis. Cell Tissue Res, 2012.

4. Suzuki, F., et al.,Regional differentiation of cell junctions in the excurrent duct epithelium of the rat testis as revealed by freeze-fracture. Anat Rec, 1978. 191(4): $\mathrm{p}$. 503-19.

5. Suzuki, F., et al.,Development of tight junctions in the caput epididymal epithelium of the mouse. Dev Biol, 1978. 63(2): p. 321-34.

6. Takano, H.,[Qualitative and quantitative histology and histogenesis of the mouse epididymis, with special emphasis on the regional difference (author's transl)]. Kaibogaku Zasshi, 1980. 55(6): p. 573-87.

7. Abe, K., et al.,Interruption of the luminal flow in the epididymal duct of the corpus epididymidis in the mouse, with special reference to differentiation of the epididymal epithelium. Arch Histol Jpn, 1984. 47(2): p. 137-47.

8. Abe, K., et al.,Ultrastructure of the mouse epididymal duct with special reference to the regional differences of the principal cells. Arch Histol Jpn, 1983. 46(1): p. 51-68.

9. Abe, K., et al.,Response of the epididymal duct in the corpus epididymidis to efferent or epididymal duct ligation in the mouse. J Reprod Fertil, 1982. 64(1): p. 69-72.

10. Zondek, L.H., et al.,Normal and abnormal development of the epididymis of the fetus and infant. Eur J Pediatr, 1980. 134(1): p. 39-44.

11. Francavilla, S., et al.,Postnatal development of epididymis and ductus deferens in the rat. A correlation between the ultrastructure of the epithelium and tubule wall, and the fluorescence-microscopic distribution of actin, myosin, fibronectin, and basement membrane. Cell Tissue Res, 1987. 249(2): p. 257-65.

12. Tajiri, S., et al.,Changes in lectin-binding sites on epididymal cells during postnatal development of the mouse. Okajimas Folia Anat Jpn, 2012. 88(4): p. 153-7.

13. Hermo, L., et al.,Epithelial cells of the epididymis show regional variations with respect to the secretion of endocytosis of immobilin as revealed by light and electron microscope immunocytochemistry. Anat Rec, 1992. 232(2): p. 202-20.

14. Shum, W.W., et al.,Plasticity of basal cells during postnatal development in the rat epididymis. Reproduction, 2013. 146(5): p. 455-69.

15. Alexander, N.J.,Prenatal development of the ductus epididymidis in the rhesus monkey. The effects of fetal castration. Am J Anat, 1972. 135(1): p. 119-34.

16. Orgebin-Crist, M.C., et al.,The effects of estradiol, tamoxifen, and testosterone on the weights and histology of the epididymis and accessory sex organs of sexually immature rabbits. Endocrinology, 1983. 113(5): p. 1703-15.

17. Robaire B, H.L., Efferent ducts, epididymis, and vas deferens: structure, functions and their regulation., in The physiology of reproduction, O.N.J. Knobil E, Editor. 1988, Raven press: New York. p. 999-1080.

18. Arrighi, S.,Primary cilia in the basal cells of equine epididymis: a serendipitous finding. Tissue Cell, 2013. 45(2): p. 140-4. 
630

631

632

633

634

635

636

637

638

639

640

641

642

643

644

645

646

647

648

649

650

651

652

653

654

655

656

657

658

659

660

661

662

663

664

665

666

667

668

669

670

671

672

673

674

19. Satir, P., et al., The primary cilium at a glance. J Cell Sci, 2010. 123(Pt 4): p. 499-503.

20. Singla, V., et al.,The primary cilium as the cell's antenna: signaling at a sensory organelle. Science, 2006. 313(5787): p. 629-33.

21. Bangs, F., et al.,Primary Cilia and Mammalian Hedgehog Signaling. Cold Spring Harb Perspect Biol, 2017. 9(5).

22. Grisanti, L., et al.,Primary cilia maintain corneal epithelial homeostasis by regulation of the Notch signaling pathway. Development, 2016. 143(12): p. 2160-71.

23. Clement, D.L., et al.,PDGFRalpha signaling in the primary cilium regulates NHE1dependent fibroblast migration via coordinated differential activity of MEK1/2ERK1/2-p90RSK and AKT signaling pathways. J Cell Sci, 2013. 126(Pt 4): p. 953-65.

24. Mohammed, S.G., et al.,Fluid shear stress increases transepithelial transport of Ca2+ in ciliated distal convoluted and connecting tubule cells. FASEB J, 2017. 31(5): p. 17961806.

25. Praetorius, H.A.,The primary cilium as sensor of fluid flow: new building blocks to the model. A review in the theme: cell signaling: proteins, pathways and mechanisms. Am J Physiol Cell Physiol, 2015. 308(3): p. C198-208.

26. Nauli, S.M., et al.,Polycystins 1 and 2 mediate mechanosensation in the primary cilium of kidney cells. Nat Genet, 2003. 33(2): p. 129-37.

27. Bangs, F.K., et al.,Lineage specificity of primary cilia in the mouse embryo. Nat Cell Biol, 2015. 17(2): p. 113-22.

28. Hildebrandt, F., et al.,Ciliopathies. N Engl J Med, 2011. 364(16): p. 1533-43.

29. Belet, U., et al.,Prevalence of epididymal, seminal vesicle, prostate, and testicular cysts in autosomal dominant polycystic kidney disease. Urology, 2002. 60(1): p. 138-41.

30. Kanagarajah, P., et al.,Male infertility and adult polycystic kidney disease--revisited: case report and current literature review. Andrologia, 2012. 44 Suppl 1: p. 838-41.

31. van der Linden, E.F., et al.,Polycystic kidney disease and infertility. Fertil Steril, 1995. 64(1): p. 202-3.

32. Frantzen, C., et al., Von Hippel-Lindau Syndrome, in GeneReviews((R)), M.P. Adam, et al., Editors. 1993: Seattle (WA).

33. Chou, A., et al.,von Hippel-Lindau syndrome. Front Horm Res, 2013. 41: p. 30-49.

34. Crespigio, J., et al.,Von Hippel-Lindau disease: a single gene, several hereditary tumors. J Endocrinol Invest, 2018. 41(1): p. 21-31.

35. Glasker, S., et al.,Epididymal cystadenomas and epithelial tumourlets: effects of VHL deficiency on the human epididymis. J Pathol, 2006. 210(1): p. 32-41.

36. Miyoshi, K., et al.,Lack of dopaminergic inputs elongates the primary cilia of striatal neurons. PLoS One, 2014. 9(5): p. e97918.

37. Miyoshi, K., et al.,Factors that influence primary cilium length. Acta Med Okayama, 2011. 65(5): p. 279-85.

38. Miyoshi, K., et al.,Lithium treatment elongates primary cilia in the mouse brain and in cultured cells. Biochem Biophys Res Commun, 2009. 388(4): p. 757-62.

39. Miyoshi, K., et al.,Pericentrin, a centrosomal protein related to microcephalic primordial dwarfism, is required for olfactory cilia assembly in mice. FASEB J, 2009. 23(10): p. 3289-97.

40. Toriyama, M., et al.,Folate-dependent methylation of septins governs ciliogenesis during neural tube closure. FASEB J, 2017. 31(8): p. 3622-3635. 
675

676

677

678

679

680

681

682

683

684

685

686

687

688

689

690

691

692

693

694

695

696

697

698

699

700

701

702

703

704

705

706

707

708

709

710

711

712

713

714

715

716

717

718

719

41. Belleannee, $\mathrm{C}$., et al.,Role of microRNAs in controlling gene expression in different segments of the human epididymis. PLoS One, 2012. 7(4): p. e34996.

42. Belleannée, C., et al.,Segmental expression of the bradykinin type 2 receptor in rat efferent ducts and epididymis and its role in the regulation of aquaporin 9. Biol Reprod, 2009. 80(1): p. 134-43.

43. Bisht, K., et al.,Dark microglia: A new phenotype predominantly associated with pathological states. Glia, 2016.

44. Araki, Y., et al.,Immortalized epididymal cell lines from transgenic mice overexpressing temperature-sensitive simian virus 40 large T-antigen gene. J Androl, 2002. 23(6): $\mathrm{p}$. 854-69.

45. Belleannée, C., et al.,Role of purinergic signaling pathways in V-ATPase recruitment to apical membrane of acidifying epididymal clear cells. Am J Physiol Cell Physiol, 2010. 298(4): p. C817-30.

46. Rueden, C.T., et al.,ImageJ2: ImageJ for the next generation of scientific image data. BMC Bioinformatics, 2017. 18(1): p. 529.

47. Chalfoun, J., et al.,MIST: Accurate and Scalable Microscopy Image Stitching Tool with Stage Modeling and Error Minimization. Sci Rep, 2017. 7(1): p. 4988.

48. Legare, C., et al.,Expression and localization of c-ros oncogene along the human excurrent duct. Mol Hum Reprod, 2004. 10(9): p. 697-703.

49. Roy, J., et al.,Tyrosine kinase-mediated axial motility of basal cells revealed by intravital imaging. Nat Commun, 2016. 7: p. 10666.

50. Shum, W.W., et al.,Transepithelial projections from basal cells are luminal sensors in pseudostratified epithelia. Cell, 2008. 135(6): p. 1108-17.

51. Domeniconi, R.F., et al.,Is the Epididymis a Series of Organs Placed Side By Side? Biol Reprod, 2016. 95(1): p. 10.

52. Dores, C., et al.,Primary cilia on porcine testicular somatic cells and their role in hedgehog signaling and tubular morphogenesis in vitro. Cell Tissue Res, 2017. 368(1): p. 215-223.

53. Tobin, J.L., et al.,The nonmotile ciliopathies. Genet Med, 2009. 11(6): p. 386-402.

54. Jerczynski, 0., et al.,Role of Dicer1-Dependent Factors in the Paracrine Regulation of Epididymal Gene Expression. PLoS One, 2016. 11(10): p. e0163876.

55. Belleannée, C., et al.,Epididymosomes Convey Different Repertoires of MicroRNAs Throughout the Bovine Epididymis. Biol Reprod, 2013.

56. Sharma, U., et al.,Biogenesis and function of tRNA fragments during sperm maturation and fertilization in mammals. Science, 2016. 351(6271): p. 391-6.

57. Joiner, A.M., et al.,Primary Cilia on Horizontal Basal Cells Regulate Regeneration of the Olfactory Epithelium. J Neurosci, 2015. 35(40): p. 13761-72.

58. Guen, V.J., et al.,EMT programs promote basal mammary stem cell and tumorinitiating cell stemness by inducing primary ciliogenesis and Hedgehog signaling. Proc Natl Acad Sci U S A, 2017.

59. Mandon, M., et al.,Isolated Rat Epididymal Basal Cells Share Common Properties with Adult Stem Cells. Biol Reprod, 2015. 93(5): p. 115.

60. Murashima, A., et al.,Essential roles of androgen signaling in Wolffian duct stabilization and epididymal cell differentiation. Endocrinology, 2011. 152(4): p. 1640-51. 
720

721

722

723

724

725

726

727

728

729

730

731

732

733

734

735

736

737

738

739

740

741

742

743

744

61. Wong, S.Y., et al.,Primary cilia can both mediate and suppress Hedgehog pathwaydependent tumorigenesis. Nat Med, 2009. 15(9): p. 1055-61.

62. Wang, K., et al.,Oncogenic Wnt/beta-catenin signalling pathways in the cancerresistant epididymis have implications for cancer research. Mol Hum Reprod, 2013. 19(2): p. 57-71.

63. Yeung, C.H., et al.,Why are epididymal tumours so rare? Asian J Androl, 2012. 14(3): p. 465-75.

64. Price, E.B., Jr.,Papillary cystadenoma of the epididymis. A clinicopathologic analysis of 20 cases. Arch Pathol, 1971. 91(5): p. 456-70.

65. Satir, P.,CILIA: before and after. Cilia, 2017. 6: p. 1.

66. Kumar, M., et al.,Epithelial Wnt/betacatenin signalling is essential for epididymal coiling. Dev Biol, 2016. 412(2): p. 234-49.

67. Wang, K., et al.,Comparison of gene expression of the oncogenic Wnt/beta-catenin signaling pathway components in the mouse and human epididymis. Asian J Androl, 2015. 17(6): p. 1006-11.

68. Koch, S., et al.,Post-transcriptional Wht Signaling Governs Epididymal Sperm Maturation. Cell, 2015. 163(5): p. 1225-36.

69. Turner, T.T., et al.,Sonic hedgehog pathway inhibition alters epididymal function as assessed by the development of sperm motility. J Androl, 2006. 27(2): p. 225-32.

70. Turner, T.T., et al.,Sonic hedgehog pathway genes are expressed and transcribed in the adult mouse epididymis. J Androl, 2004. 25(4): p. 514-22.

71. Basciani, S., et al.,Expression of platelet-derived growth factor (PDGF) in the epididymis and analysis of the epididymal development in PDGF-A, PDGF-B, and PDGF receptor beta deficient mice. Biol Reprod, 2004. 70(1): p. 168-77. 


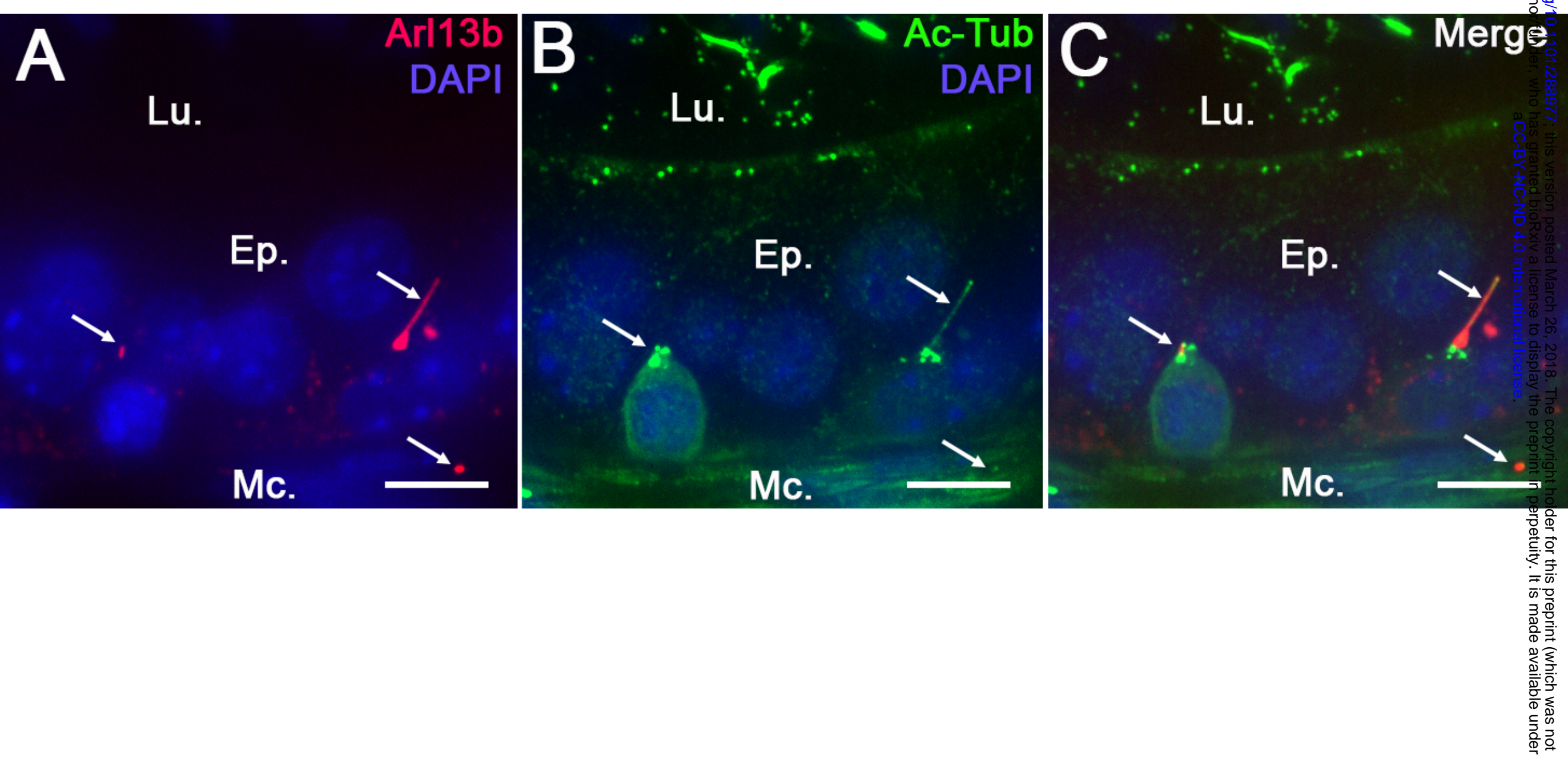




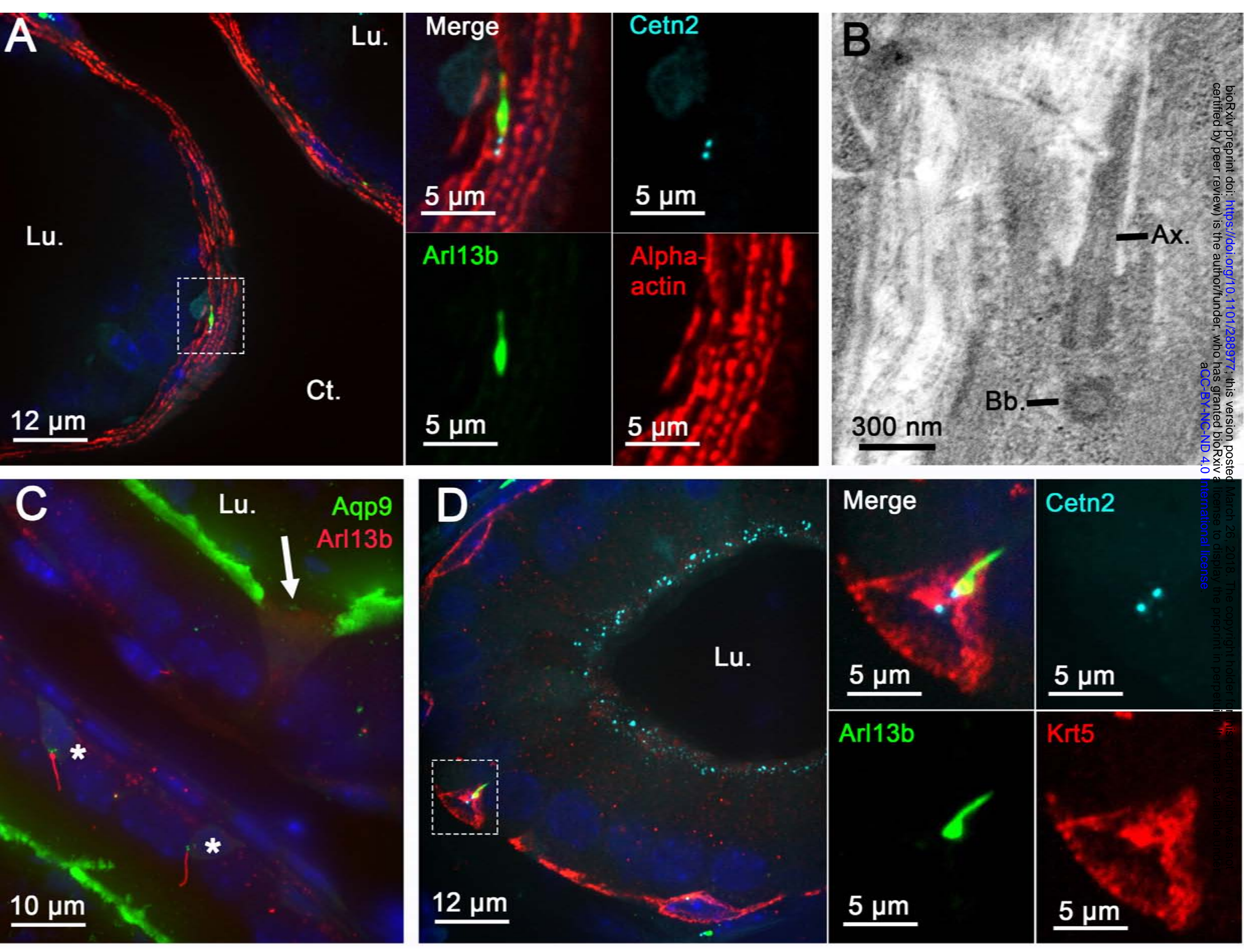


bioRxiv preprint doi: https://doi.org/10.1101/288977; this version posted March 26, 2018. The copyright holder for this preprint (which was not certified by peer review) is the author/funder, who has granted bioRxiv a license to display the preprint in perpetuity. It is made available under aCC-BY-NC-ND 4.0 International license.

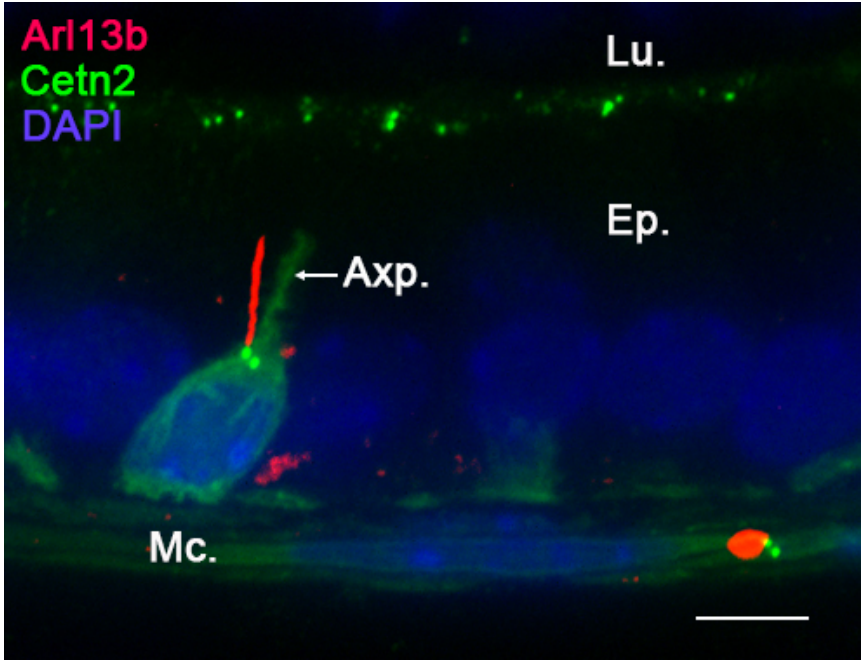



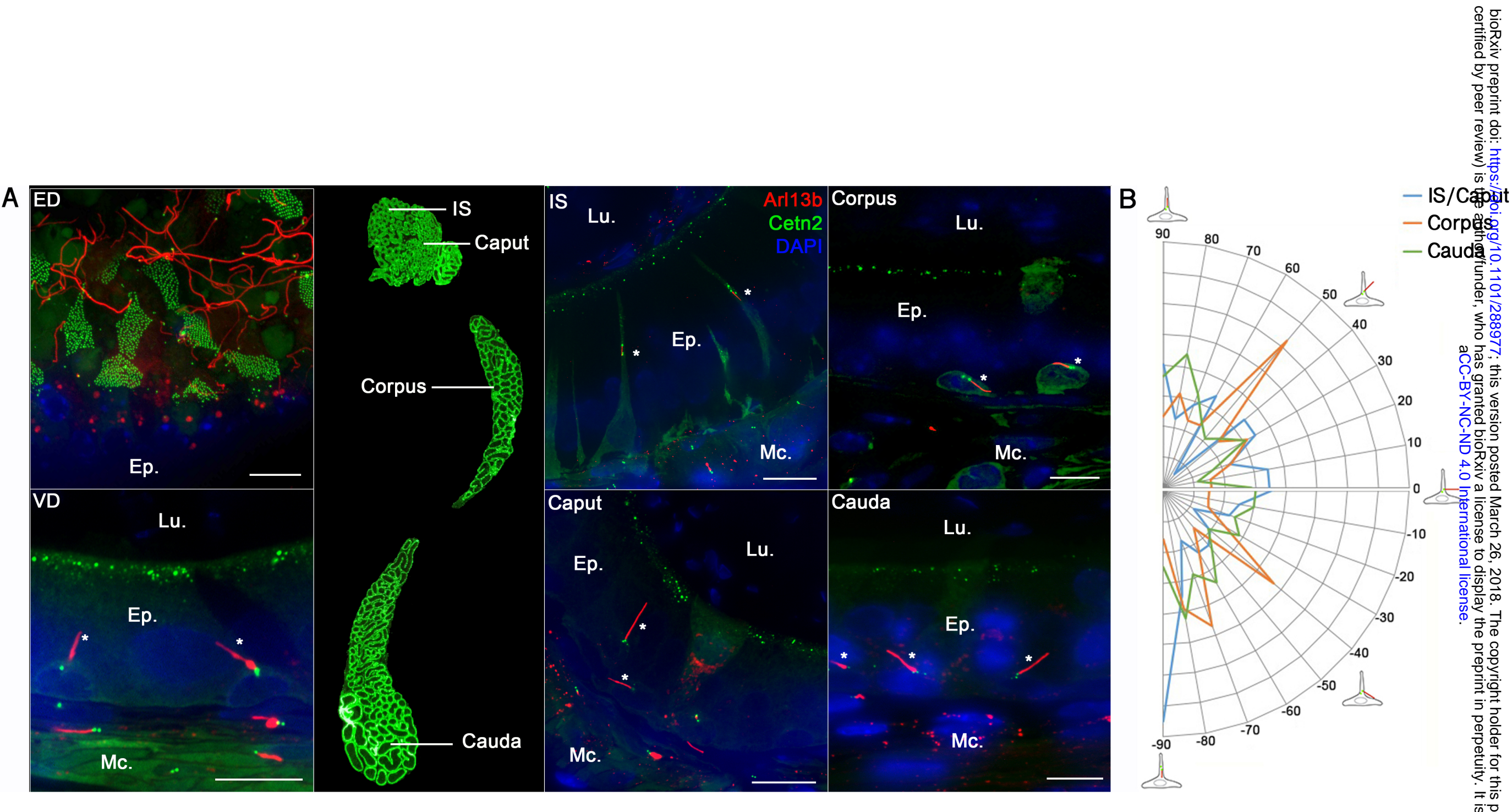


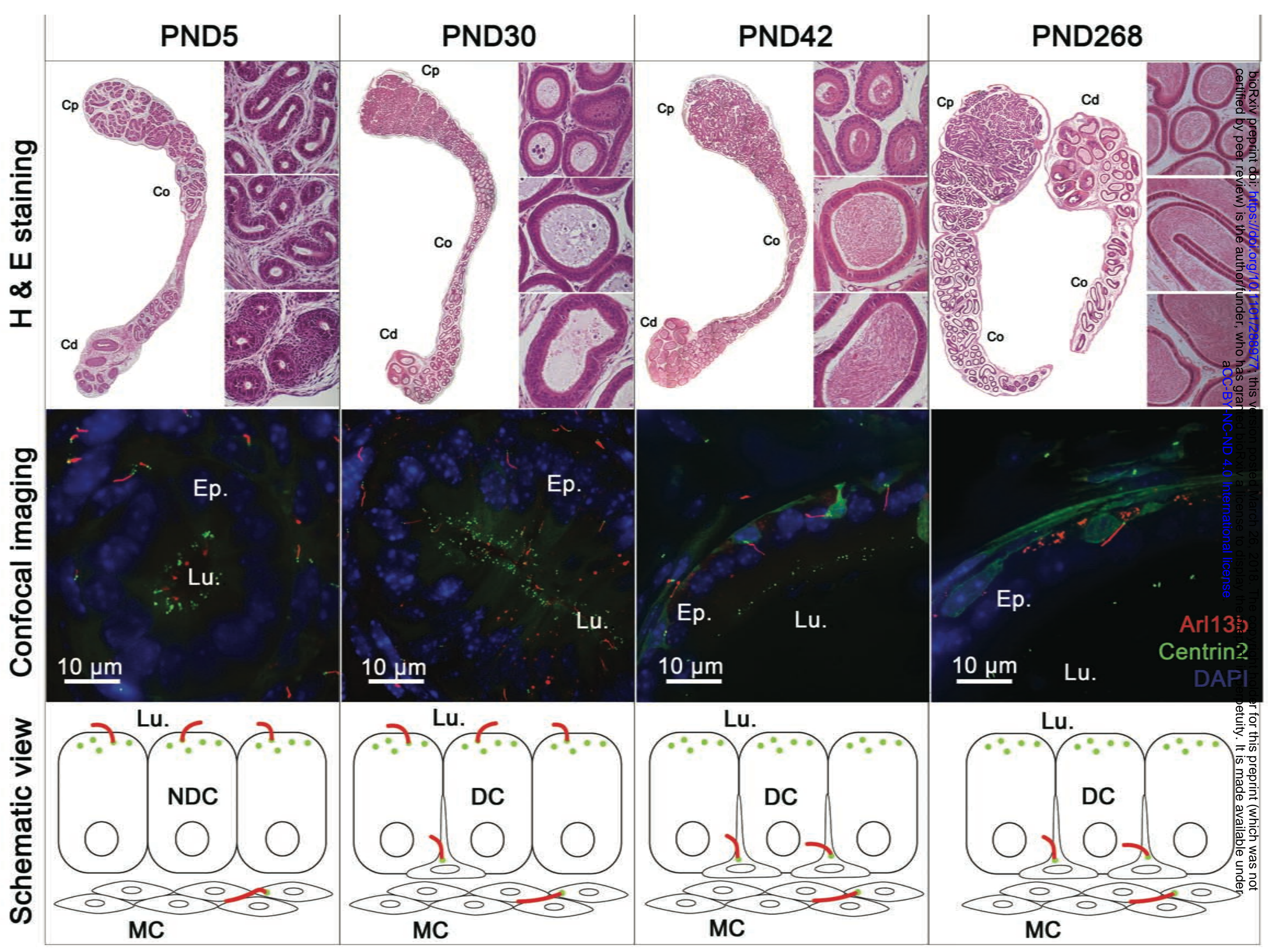


Ep.
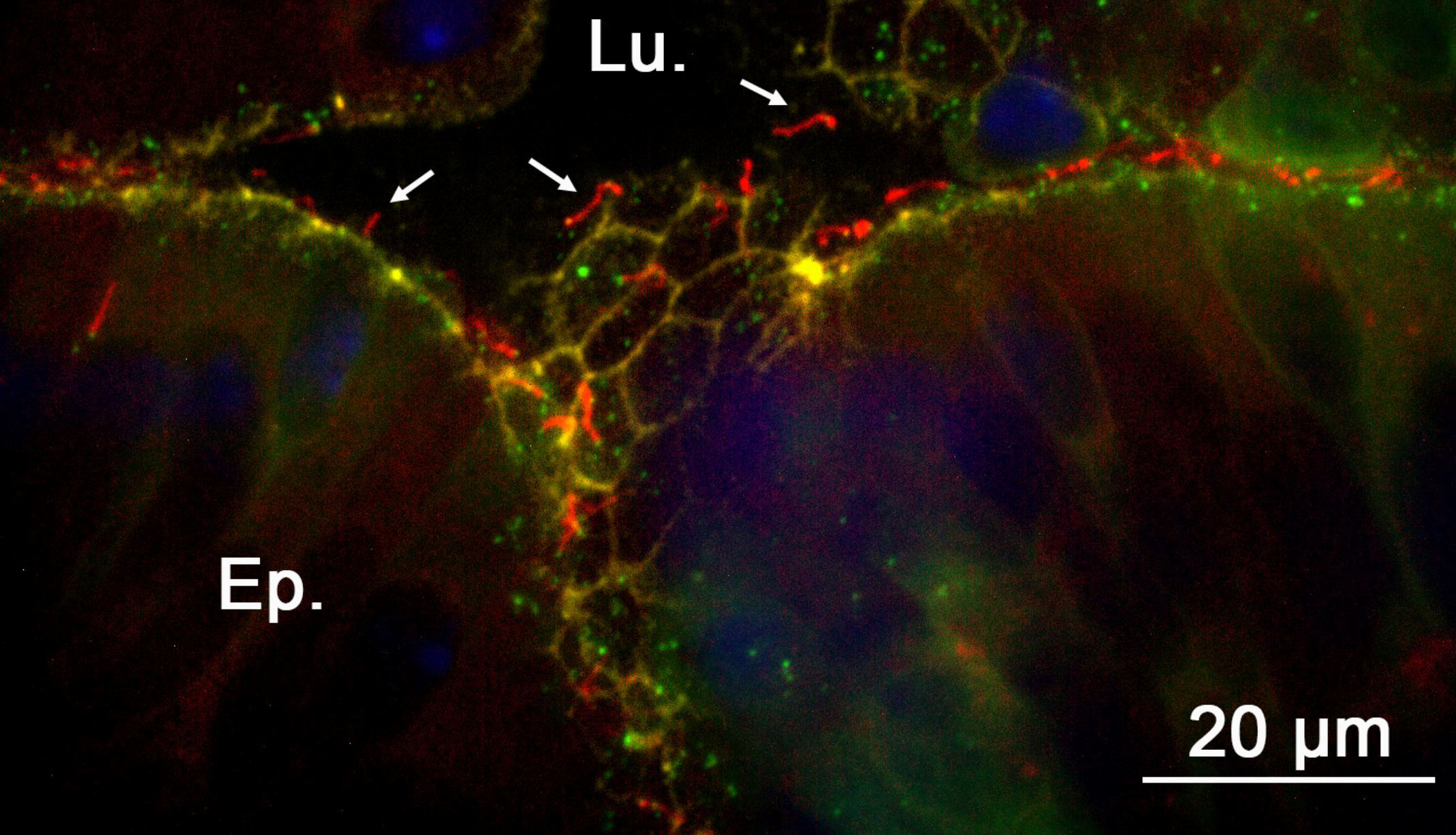

$20 \mu \mathrm{m}$ 
A $\quad$ PC1-PND7

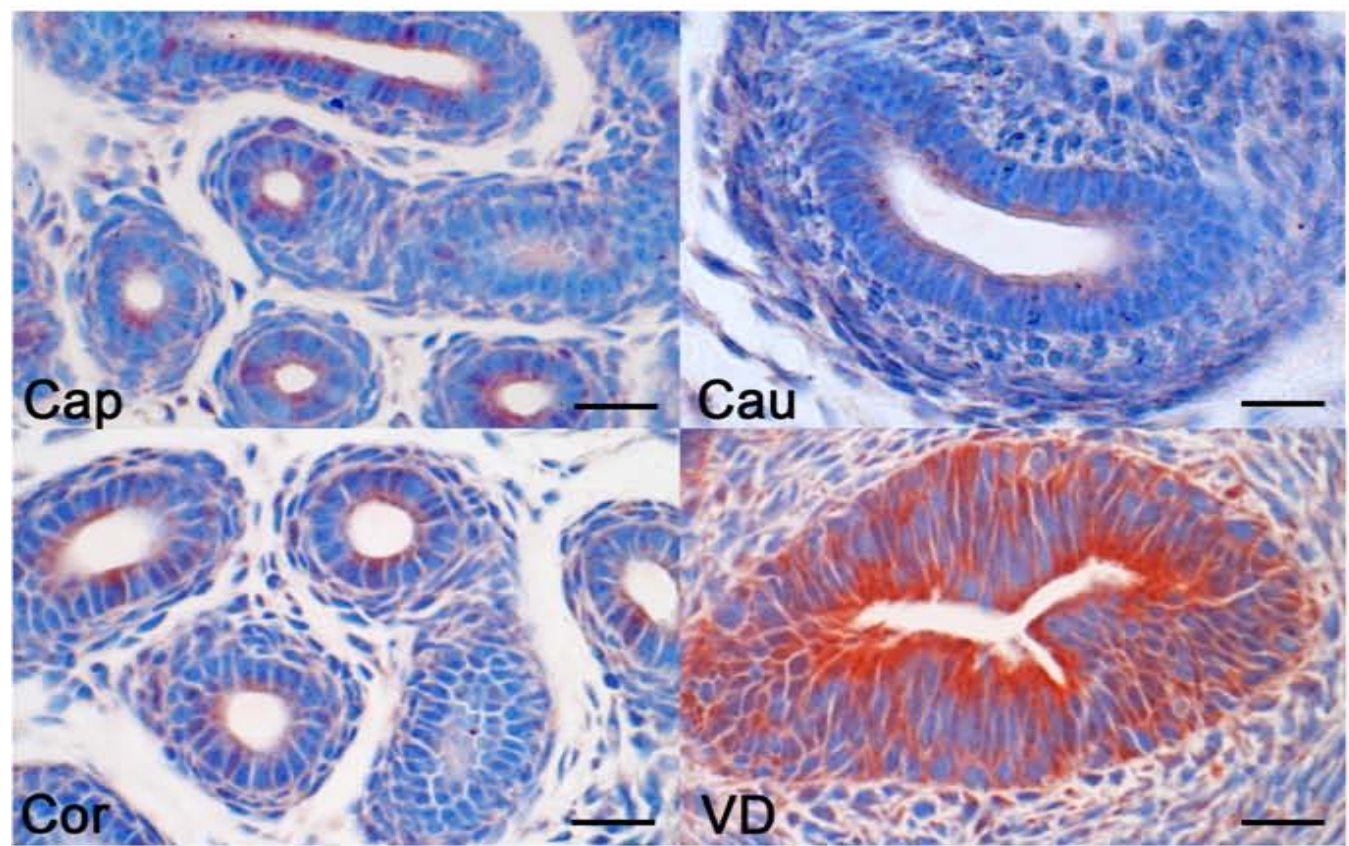

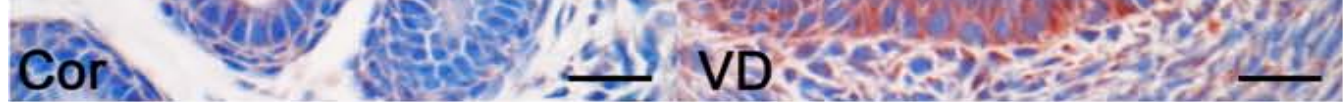

PC1-PND40

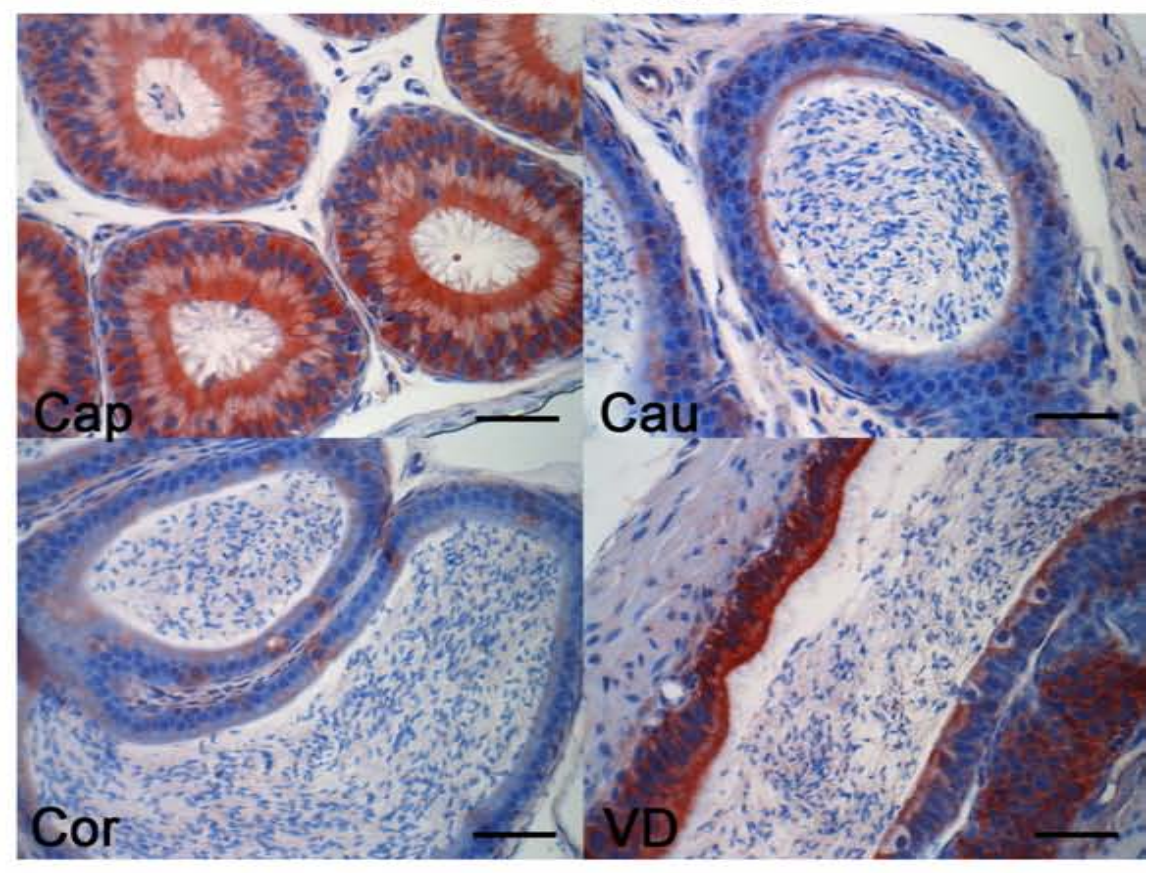

Merge

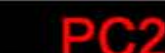

B
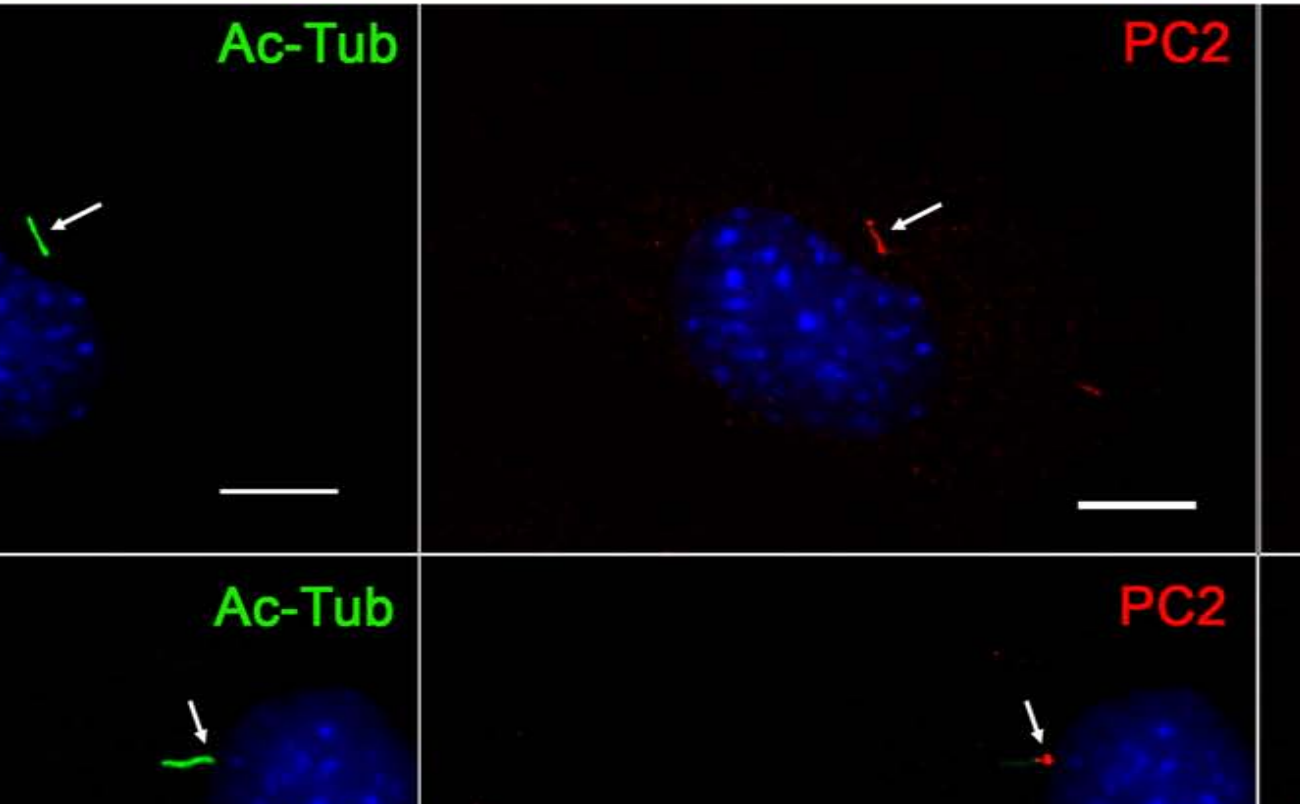

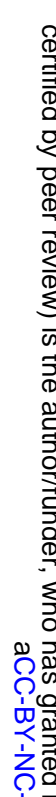

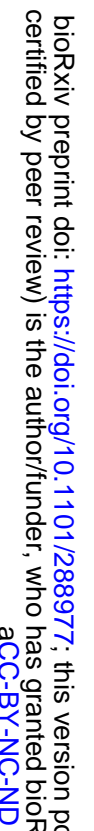

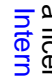

等

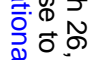

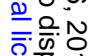

宁

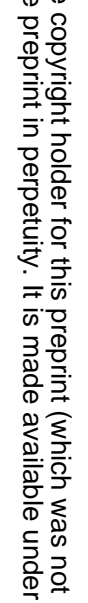


bioRxiv preprint doi: https://doi.org/10.1101/288977; this version posted March 26, 2018. The copyright holder for this preprint (which was not
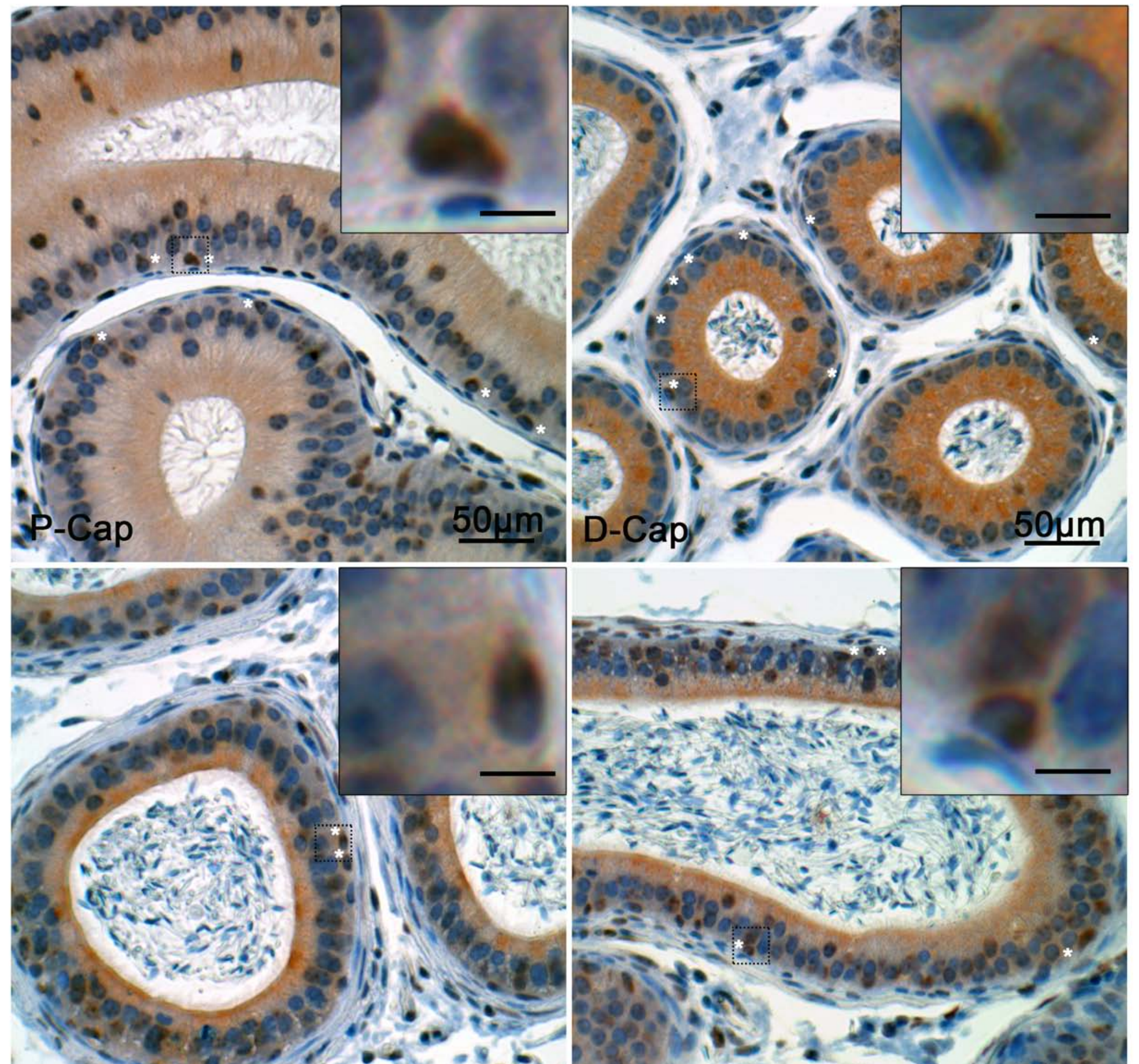

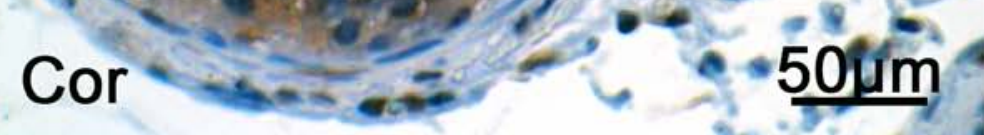

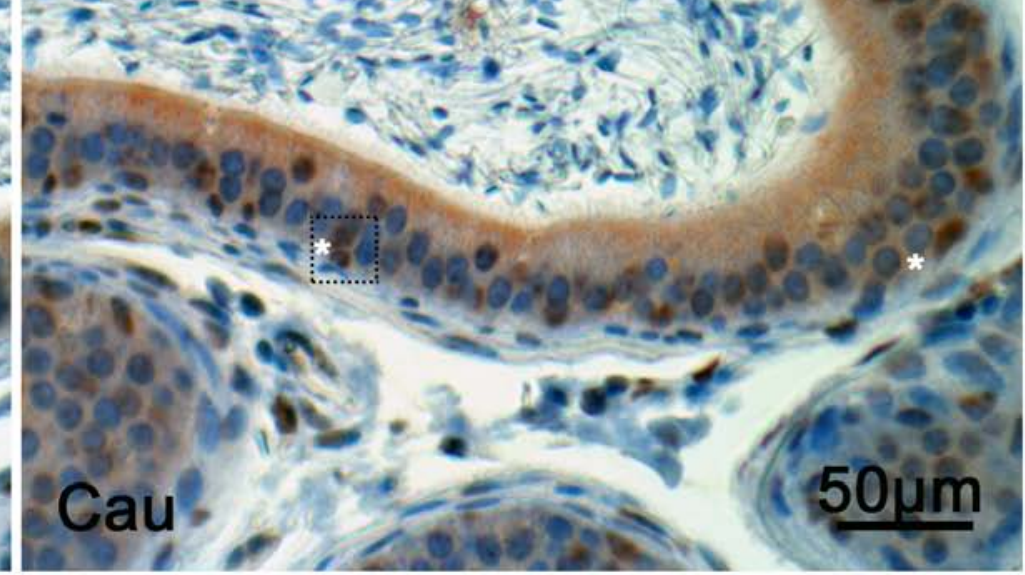




\section{Human epididymis}

Arl13b

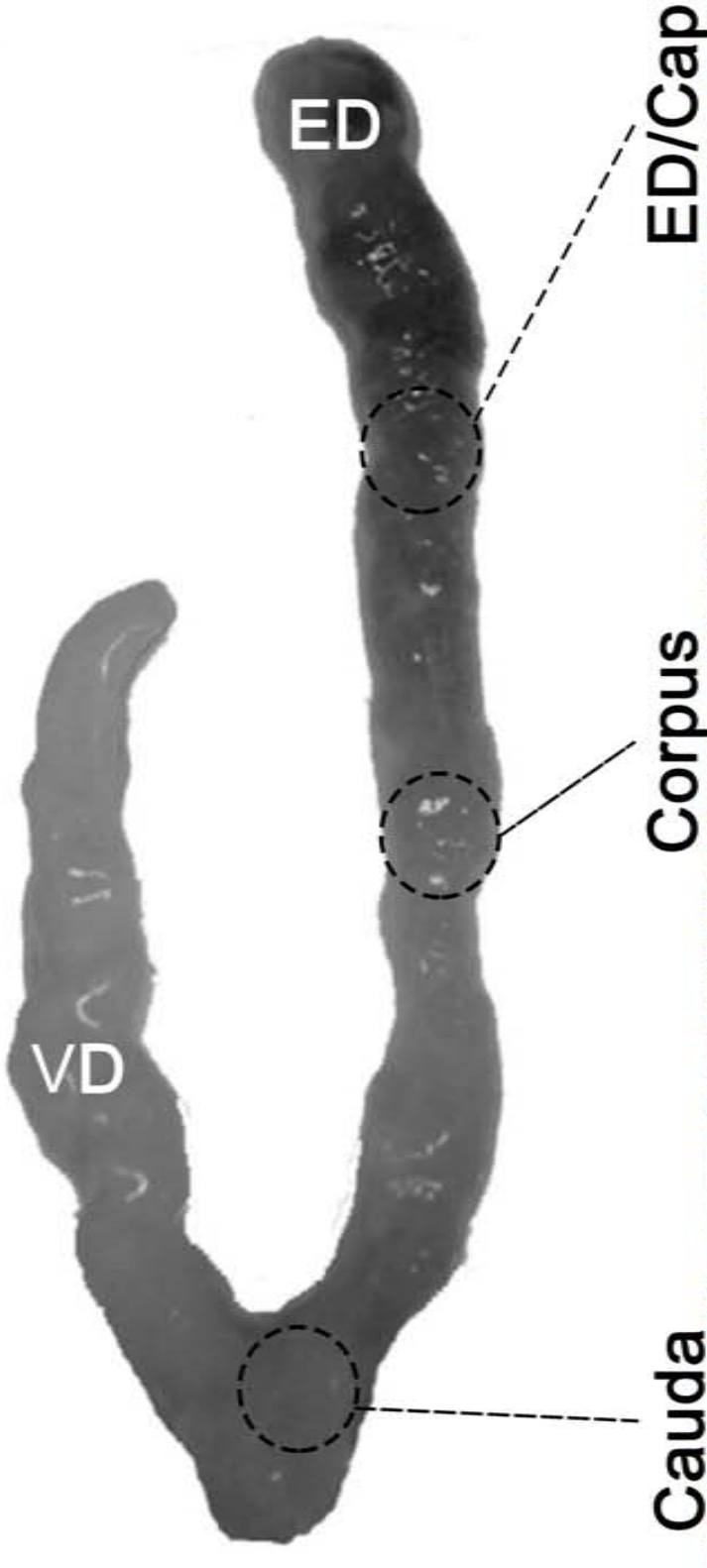

Arl13b-Inset

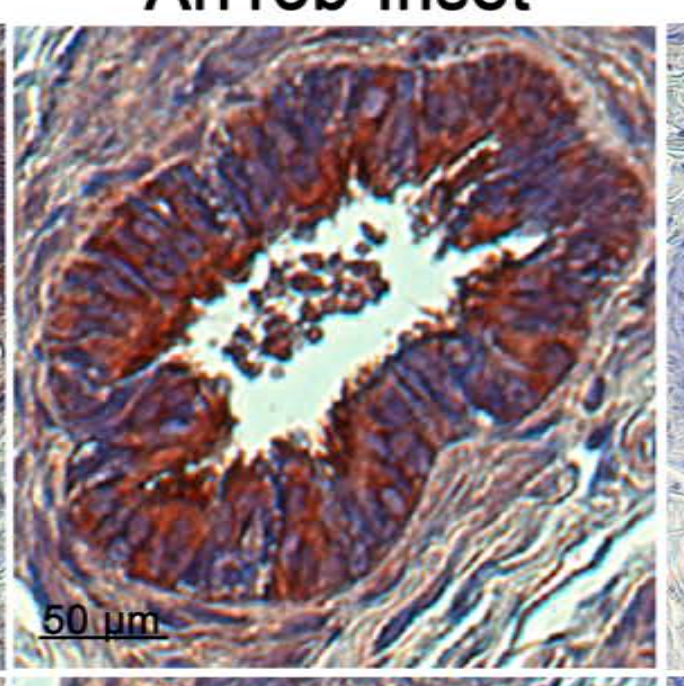

Negative control

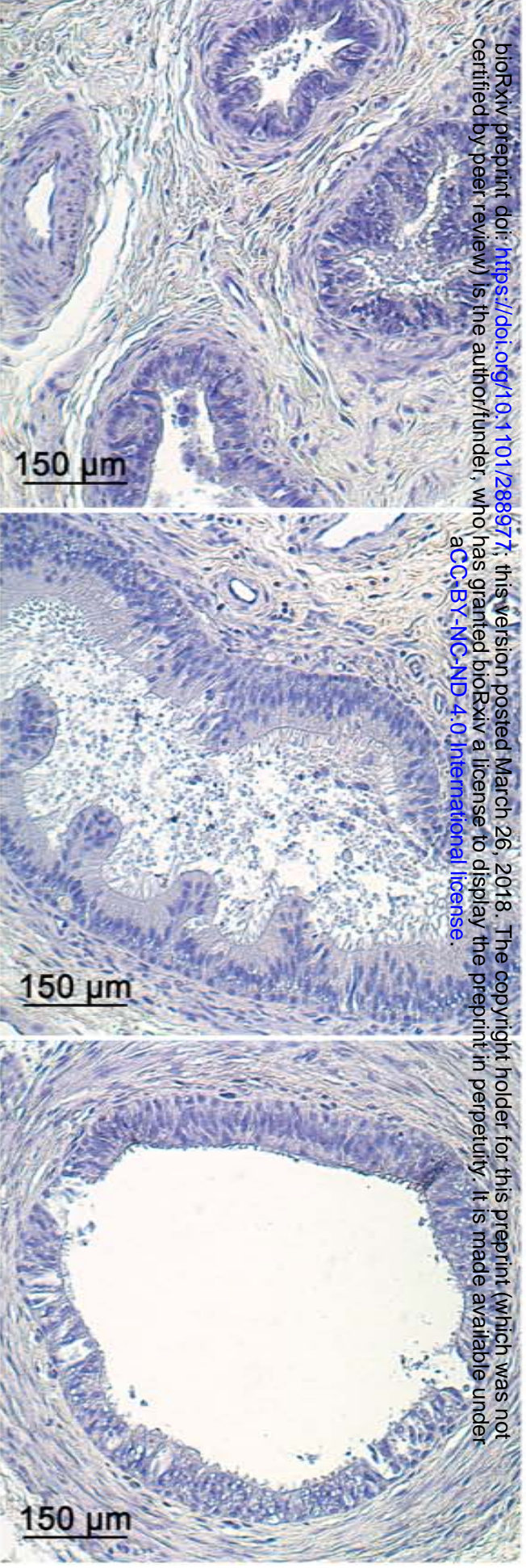




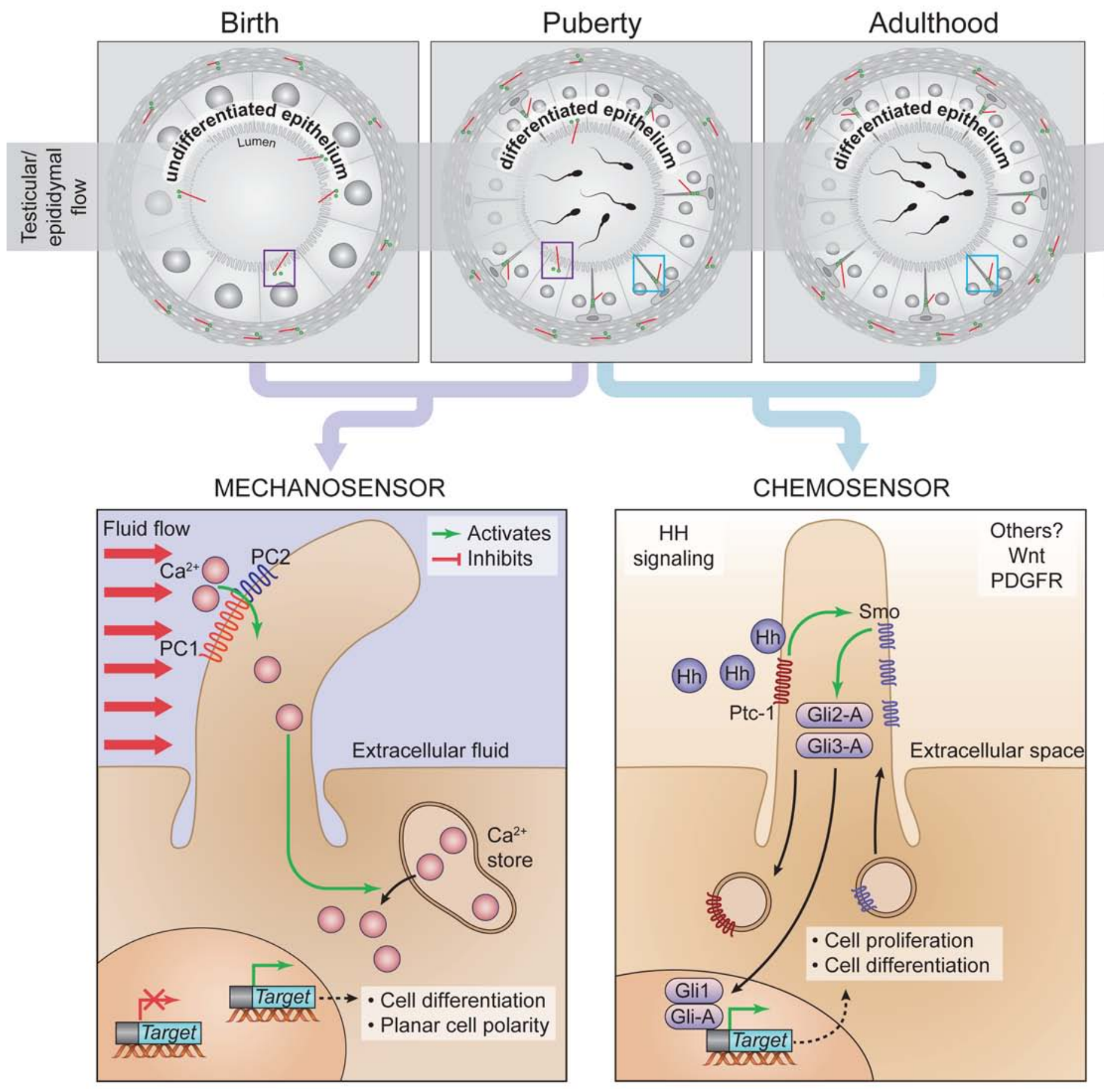

